\title{
SYNTHESIS OF STATE FEEDBACK LAWS FOR END-POINT OPTIMIZATION IN BATCH PROCESSES
}

\author{
SRINIVAS PALANKI, COSTAS KRAVARIS ${ }^{\dagger}$ and HENRY Y. WANG \\ Department of Chemical Engineering, The University of Michigan, Ann Arbor, MI 48109, U.S.A.
}

(First received 10 August 1990; accepted in revised form 17 September 1991)

\begin{abstract}
This work studies the class of singular optimal control problems, where a performance index must be optimized at the final time of operation of a batch process. Optimal state fecdback laws for the singular region of operation are derived for the first time. The existence of a singular region as well as the nature of the feedback law (static or dynamic) are completely characterized in terms of the Lie bracket structure of the system dynamics. Explicit synthesis formulae for the state feedback laws are first obtained for time-invariant systems and then extended to time-varying systems. As illustrative examples of application of the proposed methodology, we consider several end-point optimization problems in batch chemical and biochemical reactors.
\end{abstract}

\section{INTRODUCTION}

Batch and semi-batch processes are of great importance to the chemical industry. A wide variety of specialty chemicals such as antibiotics and polymers are produced in batch reactors; they are preferred due to their ease and flexibility of operation. Batch reactors are used when there are many processing steps in the chemical process, when isolation is required for reasons of sterility or safety and when the materials involved are hard to handle.

The first step to rational design of a batch chemical reactor generally consists of guaranteeing safety and operability objectives. Once these goals are achieved, the next step is to make its operation profitable. Since batch processes typically produce low-volume-highcost products, optimal operation is extremely important. Every small improvement in the process may result in considerable reduction in production costs. Batch processes are transient in nature; the process variables undergo significant changes during the batch cycle and there is no process steady state. Generally, the major objective is not to keep the system at a set point but to maximize the product of interest at the end of the batch cycle. Thus, in this situation, the role of control is to optimize a performance index at the final time rather than to regulate the process.

There is a vast body of literature dealing with the solution of end-point optimization problems on a case-by-case basis for specific batch processes. There are two different approaches that have been used.

\section{Open-loop optimization}

In this approach, the optimal trajectory of the manipulated input is first calculated off-line via optimal control theory and then implemented on the process in an open-loop fashion. There have been a plethora of articles in using this approach, primarily because of its simplicity in implementation. Hicks et al. (1969) applied Pontryagin's principle to a free-radical polymerization reactor to minimize a performance index

'Author to whom correspondence should be addressed. which was a function of deviations of conversion, number average degree of polymerization and polydispersity from their desired values. Sacks et al. (1972) used Pontryagin's principle to calculate the optimal temperature and initiator addition policy to achieve a given conversion in a free-radical polymerization batch reactor. Chen and Jeng (1978) studied batchwise bulk polymerizations operated under optimal temperature and initial initiator concentration. Thomas and Kiparissides (1984) applied Pontryagin's minimum principle to a batch polymerization reactor to calculate the optimal temperature and initiator policies that are required to produce a polymer with a desired final conversion, and desired number average and weight average molecular weights. Fishman and Biryukov (1974) considered the problem of optirıal glucose feed strategy for penicillin production. Ohno et al. (1976) used a method based on Greens' theorem for optimizing the production of lysine. Weigand (1981) calculated the optimal feeding strategy in a repeated fed-batch fermentation to maximize cell mass production.

\section{Optimal state feedback}

Using an open-loop optimization scheme is appropriate in situations where the process model is accurately known and there are no external disturbances to the process. To account for unanticipated changes in the process or the environment, the use of optimal state feedback has been proposed in the literature in a number of specific applications. In this approach, the solution to the optimization problem is calculated off-line as a function of the states and implemented as a state feedback law. In this way, the process is "forced" to follow a path in state space which has been determined a priori. Modek and Lim (1987) used this approach to show that the closed-loop system was able to attenuate errors in initial conditions in lysine fermentation. Chen and Huang (1981) derived the optimal initiator feeding policy as a function of the system states in a semi-batch isothermal solution polymerization process. 
The derivation of optimal state feedback laws is available in the literature only for specific batch applications. There are no results available which provide conditions under which a state feedback exists or general formulae for the control law. The purpose of this paper is to study the general problem of synthesizing optimal state feedback laws which guarantee optimality at a fixed final time of the batch. We first give a brief review of the optimal control formulation of the end-point optimization problem both in the context of the classical Pontryagin's principle as well as from the modern geometric perspective. The latter provides the notion of degree of singularity that allows a more transparent characterization of the necessary conditions for optimality. We then proceed to the main mission of this paper, which is to derive for the first time, optimal state feedback laws for the singular region of operation. Time-invariant systems are studied first. Theorem 1 provides a complete characterization of the case of infinite degree of singularity; it is shown that either a singular extremal does not exist or, when it does, the end-point optimization problem reduces to a standard regulator problem. Theorems 2 and 3 study the case of finite degree of singularity; they provide explicit state feedback laws for optimality at the end of the batch time. All the results are then extended to include time-varying systems (via theorems 4 and 5). Once we have derived state feedback laws for end-point optimization, we discuss some issues on how these laws can be implemented as part of a closed-loop scheme. Finally, as illustrative examples of the application of the proposed methodology, we consider several end-point optimization problems in batch chemical and biochemical reactors.

\section{END-POINT OPTIMIZATION FOR TIME-INVARIANT} SYSTEMS

Formulation of the end-point optimization problem: the classical optimal control perspective

For a large class of chemical processes, the manipulated input appears linearly in the state model. For such systems, end-point optimization problems can be mathematically formulated as follows. Minimize the performance index

$$
J=\phi\left(x\left(t_{f}\right)\right)
$$

subject to the dynamics

$$
\begin{aligned}
\dot{x} & =f(x)+g(x) u, \quad 0 \leqslant t \leqslant t_{s} \\
x(0) & =x_{0}
\end{aligned}
$$

where $u$ is the (scalar) manipulated input, $x$ is the $n$-vector of states, $t_{f}$ is the final time, $f(x)$ and $g(x)$ are smooth vector functions and $\phi(x)$ is a smooth scalar function such that

$$
\begin{aligned}
g(x) & \neq\left[\begin{array}{llll}
0 & 0 & \ldots & 0
\end{array}\right] \\
\frac{\partial \phi}{\partial x}(x) & \neq\left[\begin{array}{llll}
0 & 0 & \ldots & 0
\end{array}\right]
\end{aligned}
$$

for all $x$.
By Pontryagin's principle, the above minimization problem is equivalent to minimizing the Hamiltonian

$$
H(x, \lambda, u)=\lambda^{T} f(x)+\lambda^{T} g(x) u
$$

where $\lambda$ is the solution of

$$
\begin{aligned}
\dot{\lambda}^{T} & =-\lambda^{T}\left(\frac{\partial f}{\partial x}(x)+\frac{\partial g}{\partial x}(x) u\right) \\
\lambda\left(t_{f}\right) & =\frac{\partial \phi}{\partial x}\left(x\left(t_{f}\right)\right) .
\end{aligned}
$$

Because the Hamiltonian is linear in the control, $u$, the optimal control problem is singular. The optimal trajectories $u(t)$ will correspond to either hitting a constraint or to a singular extremal. By definition, a function $u(t)$ is called a singular extremal if it satisfies

$$
H_{u}(x, \lambda, u)=0
$$

and in addition

$$
H_{u u}(x, \lambda, u)=0 .
$$

Methods for calculating singular extremals are available in the classical optimal control literature [see standard texts such as Bryson and Ho (1975), Denn (1969) and Ray (1981)]. In the above optimization problem,

$$
H_{u}(x, \lambda, u)=\lambda^{T} g(x) .
$$

For a singular $u(t)$ we will have

$$
\lambda^{T} g(x)=0
$$

for all times; therefore its successive time derivatives are also zero. Thus, we have

$$
\begin{aligned}
& \frac{\mathrm{d}}{\mathrm{d} t}\left(\lambda^{T} g(x)\right)=0 \\
& \frac{\mathrm{d}^{2}}{\mathrm{~d} t^{2}}\left(\lambda^{T} g(x)\right)=0
\end{aligned}
$$

This sequence of differentiations is performed until $u$ appears explicitly. The resulting expression can then be solved for $u$ in terms of $x$ and $\lambda$.

The function $\lambda^{T} g(x)$ is called the switching function. This function vanishes over the singular time interval. Outside the singular interval, the manipulated input, $u$, takes either its maximum value, $u_{\max }$, or its minimum value, $u_{\min }$ depending on the sign of the switching function. When $\lambda^{T} g(x)<0, u=u_{\max }$ and when $\lambda^{T} g(x)>0, u=u_{\text {min }}$. The time(s) at which the input switches from singular to nonsingular interval and vice versa or the time(s) at which the input switches from minimum to maximum value and vice versa is called switching time.

Gabasov and Kirillova (1972) have reviewed the higher-order necessary conditions for optimality. These conditions distinguish the optimal controls from nonoptimal controls which satisfy the first-order conditions. A second-order necessary condition for 
optimality, called the generalized Legendre-Clebsch condition, can be stated as follows:

$(-1)^{k} \frac{\partial}{\partial u}\left(\left(\frac{\mathrm{d}}{\mathrm{d} t}\right)^{2 k} H_{u}(x, \lambda, u)\right) \geqslant 0, \quad k=0,1,2 \ldots$

This condition was originally developed by Kelley et al. (1967). Third-order necessary conditions for optimality have also been developed for singular problems (Krener, 1977; Lamnabhi-Lagarrigue and Stefani, 1990).

Characterization of singular extremals: a geometric perspective

The previous subsection gave an overview of the classical optimal control formulation. In the present subsection it will be seen that geometric tools can be used to obtain a more concrete and transparent representation of the necessary conditions for optimality. These results will be used in the next subsection to develop, for the first time, a general framework for end-point optimal state feedback synthesis.

In the classical approach, the optimal control is determined by solving the algebraic equations which result by successively differentiating the switching function $H_{u}(x, \lambda, u)$ until the control, $u$, appears explicitly. This gives rise to the concept of order or degree of a singular problem, originally introduced by Kopp and Moyer (1965). Since then, problemoriented definitions of order have been suggested (Kelley et al., 1967; Robbins, 1967; McDanell and Powers, 1971) and the issue of defining the order of the singular control problem became a subject of controversy. Lewis (1980) surveys the various definitions of singular control order. The issue of defining the order of a singular control problem was resolved in the work of Krener (1977). In the same paper, Krener developed a higher-order minimum principle and elucidated the importance of Lie brackets in singular optimal control problems.

We first review the definition of Lie brackets of a nonlinear system:

Given $\mathrm{f}(\mathrm{x}), \mathrm{g}(\mathrm{x}) \mathrm{C}^{\infty}$ vector fields on $\mathbb{R}^{\mathrm{n}}$, the Lie bracket $[\mathrm{f}, \mathrm{g}](\mathrm{x})$ is a vector field defined by

$$
[f, g](x)=\frac{\partial g(x)}{\partial x} f(x)-\frac{\partial f(x)}{\partial x} g(x)
$$

where $\partial \mathrm{f}(\mathrm{x}) / \partial \mathrm{x}$ and $\partial \mathrm{g}(\mathrm{x}) / \partial \mathrm{x}$ are the Jacobians. $[\mathrm{f}, \mathrm{g}]$ is also a $\mathrm{C}^{\infty}$ vector field on $\mathbb{R}^{\mathrm{n}}$. One can define iterated Lie brackets $[f,[f, g]],[f,[f,[f, g]]]$ etc. The following notation is standard:

$$
\begin{aligned}
a d_{f}^{0} g(x) & =g(x) \\
a d_{f}^{1} g(x) & =[f, g](x) \\
a d_{f}^{2} g(x) & =[f,[f, g]](x) \\
& \vdots \\
a d_{f}^{k} g(x) & =\left[f, a d_{f}^{k-1} g\right](x) .
\end{aligned}
$$

The system theoretic significance of the Lie brackets can be illustrated by the following well-known result.

Proposition 1 (Hunt, 1982): The dynamic system (2) is locally controllable if the vector fields $\mathrm{g}(\mathrm{x})$, $\operatorname{ad}_{\mathrm{f}}^{1} \mathrm{~g}(\mathrm{x}), \ldots, \mathrm{ad}^{-1} \mathrm{~g}(\mathrm{x})$ are linearly independent locally for every $x$.

Throughout this section, we will make the standing assumption that $g(x), a d\} g(x), \ldots, a d_{f}^{n-1} g(x)$ are linearly independent almost everywhere in $x$ to guarantee local controllability of the system almost everywhere. This assumption is equivalent to

$$
\operatorname{det}\left[\begin{array}{llll}
g(x) & a d_{f}^{1} g(x) & \ldots & a d_{f}^{n-1} g(x)
\end{array}\right] \neq 0 .
$$

Uncontrollable situations where some of these vector fields are linearly dependent for every $x$ can be handled as will be illustrated in examples 2 and 3 .

Definition 1: The system governed by equation (2) has a finite degree of singularity $s$ if

$$
\begin{array}{r}
{\left[g, a d_{f}^{v} g\right](x) \in \operatorname{span}\left\{g(x), a d_{f}^{1} g(x), \ldots, a d_{f}^{v} g(x)\right\},} \\
\text { for all } v \leqslant s-1
\end{array}
$$

$\left[g, a d_{f}^{s} g\right](x) \notin \operatorname{span}\left\{g(x), a d_{f}^{1} g(x), \ldots, a d_{f}^{s} g(x)\right\}$.

The system governed by eq. (2) has an infinite degree of singularity $(s=\infty)$ if

$$
\begin{array}{r}
{\left[g, a d_{f}^{v} g\right](x) \in \operatorname{span}\left\{g(x), a d_{f}^{1} g(x), \ldots, a d_{f}^{v} g(x)\right\},} \\
\text { for all } v \geqslant 0 .
\end{array}
$$

Since $g(x), a d_{f}^{1} g(x), \ldots, a d_{f}^{n-1} g(x)$ are assumed to be linearly independent, it follows from the definition that $1 \leqslant s \leqslant(n-2)$ or $s=\infty$.

Remark 1: It can be shown that this definition is completely equivalent to Krener's definition of degree of singularity (Krener, 1977) for the class of systems that have $g(x), a d_{f}^{1} g(x), \ldots, a d_{f}^{n-1} g(x)$ linearly independent. Though the latter does not have this restriction, it is more difficult to use.

The following proposition is a rather straightforward consequence of known results [see Gabasov and Kirillova (1972)] when the above definition of $s$ is used.

Proposition 2: Consider a system governed by eq. (2) with finite degree of singularity $\mathrm{s}$. Then $\mathrm{s}$ is odd and the first-order necessary conditions for optimality for a singular extremal can be written as follows:

$$
\begin{gathered}
\lambda^{T} g(x)=0 \\
\lambda^{T} a d_{f}^{1} g(x)=0 \\
\lambda^{T} a d_{f}^{2} g(x)=0 \\
\vdots \\
\lambda^{T} a d_{f}^{s} g(x)=0 \\
\lambda^{T} a d_{f}^{s+1} g(x)+\lambda^{T}\left[g, a d_{f}^{s} g\right](x) u=0
\end{gathered}
$$


where $\lambda^{T}$ is the solution of eq. (5). Furthermore, the second-order necessary condition for optimality takes the form

$$
(-1)^{(s+1) / 2} \lambda^{T}\left[g, a d_{f}^{s} g\right](x) \geqslant 0 .
$$

If eq. (2) has an infinite degree of singularity, then the first-order necessary conditions for optimality take the form $\lambda^{\mathrm{T}} \operatorname{ad}_{\mathbf{r}}^{v} \mathbf{g}(\mathbf{x})=0$ for all $\boldsymbol{v} \geqslant 0$.

Singular extremal as a state feedback law independent of the adjoint states

The previous subsections provided a brief review of the classical as well as the geometric perspective of singular optimal control theory. It was seen in proposition 2 that the first-order and second-order necessary conditions for optimality in the singular region can be put in a very concrete form in terms of the system Lie brackets. The key difficulty in using these necessary conditions arises from the presence of the adjoint states. To be able to calculate the optimal control input, $u(t)$, one must solve a two-point boundary value problem that involves forward-in-time integration of the state equations and backward-in-time integration of the adjoint equations. The analysis of the optimality conditions would be greatly facilitated if the adjoint states could be eliminated from the necessary conditions. This elimination problem was studied in a general (not necessarily singular) setting in the work of Rouff and Lamnabhi-Lagarrigue (1986).

In the present subsection we will address for the first time the problem of synthesizing state feedback laws to track singular trajectories, based on eliminating the adjoint states from the first-order necessary conditions for optimality. The case $s=\infty$ will be studied first; theorem 1 will show that the problem of tracking singular extremals can be formulated as a standard regulator problem whenever a singular extremal exists. In theorem 2, we will derive a static state feedback law that tracks the singular extremal when the degree of singularity is equal to $(n-2)$. Finally, in theorem 3, we will derive a dynamic state feedback law that tracks the singular trajectories when the degree of singularity is less than $(n-2)$.

Theorem 1: Consider a system of the form of eq. (2) with degree of singularity $\mathrm{s}=\infty$ and a performance index of the form of eq. (1).

(i) If $\mathrm{g}(\mathrm{x}), \operatorname{ad}_{\mathrm{f}}^{1} \mathrm{~g}(\mathrm{x}), \ldots, \operatorname{ad}_{\mathrm{f}}^{\mathrm{n}-1} \mathrm{~g}(\mathrm{x})$ are linearly independent for all $x$, then a singular extremal does not exist.

(ii) If $\mathrm{g}(\mathrm{x}), \operatorname{ad}_{\mathrm{f}}^{1} \mathrm{~g}(\mathrm{x}), \ldots, \operatorname{ad}_{\mathrm{f}}^{\mathrm{n}-1} \mathrm{~g}(\mathrm{x})$ are linearly independent almost everywhere, then the state trajectories corresponding to a singular extremal evolve on the surface $\mathbf{S}(\mathrm{x})=0$ where linear independence of $\mathbf{g}(\mathrm{x})$, $\operatorname{ad}_{\mathrm{f}}^{1} \mathrm{~g}(\mathrm{x}), \ldots, \operatorname{ad}_{\mathrm{f}}^{\mathrm{n}-1} \mathrm{~g}(\mathrm{x})$ is lost:

$$
S(x)=\operatorname{det}\left[\begin{array}{llll}
g(x) & a d_{f}^{1} g(x) & \ldots & a d_{f}^{n-1} g(x)
\end{array}\right]=0 .
$$

Proof of (i): We prove this part of the theorem by contradiction. Let us assume that a singular extremal exists for the system (2) with degree of singularity $s=\infty$. The first-order necessary conditions on the singular extremal are as follows:

$$
\begin{aligned}
\lambda^{T} g(x) & =0 \\
\lambda^{T} a d_{f}^{1} g(x) & =0 \\
\lambda^{T} a d_{f}^{2} g(x) & =0 \\
\vdots & \\
\lambda^{T} a d_{f}^{n-1} g(x) & =0
\end{aligned}
$$

The first $n$ equations can be written in compact form as

$$
\lambda^{T}\left[g(x) \quad a d_{f}^{1} g(x) \quad \ldots \quad a d_{f}^{n-1} g(x)\right]=0 .
$$

Since $\lambda(t)$ is an absolutely continuous nonzero vector function in $\mathbb{R}^{n}$, it follows that

$$
\operatorname{det}\left[g(x) \quad a d_{f}^{1} g(x) \quad \ldots \quad a d_{f}^{n-1} g(x)\right]=0
$$

for all $x$ in some nonempty set in $\mathbb{R}^{n}$. This contradicts the assumption of glubal linear independence of $g(x)$, $\operatorname{ad}_{f}^{1} g(x), \ldots, \operatorname{ad}_{f}^{n-1} g(x)$.

Proof of (ii): The first-order necessary conditions on the singular extremal are given by eq. (16). Using exactly the same argument as in the proof of (i), we conclude that the state trajectories corresponding to the singular control must lie on the surface

$$
S(x)=0 \text {. }
$$

Corollary 1: Controllable linear systems do not possess singular extremals.

Proof: Consider a single-input controllable linear system of the form

$$
\dot{x}=A x+b u \text {. }
$$

For this system $a d_{f}^{k} g(x)=(-1)^{k} A^{k} b$ for all $k=0$, $1,2, \ldots$ Hence,

$$
\left[g, a d_{f}^{k} g\right](x)=0 \text { for all } k=0,1,2, \ldots
$$

Thus, $s=\infty$. Since the system under consideration is controllable for all $x$, the vector fields defined by $a d_{f}^{k} g(x)$ for $k=0,1,2, \ldots,(n-1)$ are linearly independent. Thus, from theorem 1 , the system under consideration does not possess a singular extremal.

Corollary 2: If the vector fields $\mathrm{g}(\mathbf{x}), \operatorname{ad}_{f}^{1} \mathbf{g}(\mathbf{x}), \ldots$, $\operatorname{ad}_{f}^{n-1} \mathrm{~g}(\mathrm{x})$ are linearly independent for all $\mathrm{x}$ and the set of vector fields

$$
\left\{g(x), a d_{f}^{1} g(x), \ldots, a d_{f}^{n-2} g(x)\right\}
$$

is involutive for all $x$, i.e. the system is feedback linearizable in the Hunt-Su-Meyer sense (Hunt et al., 1983), then such a system does not possess a singular extremal. 
Proof: From the involutivity assumption, for all $k=0,1, \ldots,(n-2)$

$$
\left[g, a d_{f}^{k} g\right](x)=\sum_{j=0}^{n-2} \alpha_{j k}(x) a d_{f}^{k} g(x)
$$

where $\alpha_{j k}(x)$ are scalar fields in $x$.

Hence, $s>(n-2)$. But $s \leqslant(n-2)$, if finite. Thus, $s=\infty$. Since the vector fields defined by $a d_{f}^{k} g(x)$ for $k=0,1,2, \ldots,(n-1)$ are linearly independent for all $x$, from theorem 1 the system under consideration does not possess a singular extremal.

When a singular extremal does not exist, the optimal control is bang-bang in nature, that is, the optimal control corresponds to the maximum or the minimum value of the manipulated input. Since controllable linear systems and feedback-linearizable nonlinear systems do not possess singular extremals, the existence of singular extremals is a manifestation of truly nonlinear behavior. Consequently, if it is known on physical grounds that a singular extremal exists for a given process, modeling such a process by a linear model or an involutive model is inappropriate for optimization purposes.

Another interesting conclusion from theorem 1 is that optimality may correspond to loss of state controllability. In this case, the optimal state trajectories must lie on the surface $S(x)=0$ [eq. (15)] and the problem of synthesizing a state feedback to track the singular cxtremals reduces to a standard regulation problem for the output

$$
y=S(x)
$$

with zero set point. This regulation problem can be solved by using standard techniques as long as $S(x)$ has a finite relative order. For instance, in the case of unity relative order $\{(\partial S(x) / \partial x) g(x) \neq 0\}$

$$
u=-\frac{(\partial S(x) / \partial x) f(x)+\beta S(x)}{(\partial S(x) / \partial x) g(x)}
$$

where $\beta$ is an adjustable positive parameter, is an appropriate feedback law for the above regulation problem.

Theorem 2: For a system of the form of eq. (2) and a performance index of the form of eq. (1), the singular trajectories can be tracked by static state feedback when $s=(n-2)$. The optimal state feedback law is given by

$u=-\frac{\operatorname{det}\left[g(x) a d_{f}^{1} g(x) \ldots a d_{f}^{n-1} g(x)\right]}{\operatorname{det}\left[g(x) a d_{f}^{1} g(x) \ldots a d_{f}^{n-2} g(x)\left[g, a d_{f}^{n-2} g\right](x)\right]}$

Proof. The first-order necessary conditions for $s=(n-2)$ can be written as

$$
\begin{gathered}
\lambda^{\mathbf{T}} g(x)=0 \\
\lambda^{\mathbf{T}} a d_{f}^{1} g(x)=0 \\
\vdots \\
\lambda^{T} a d_{f}^{n-2} g(x)=0 \\
\lambda^{T} a d_{f}^{n-1} g(x)+\lambda^{T}\left[g, a d_{f}^{n-2} g\right](x) u=0 .
\end{gathered}
$$

Since these are $\boldsymbol{n}$ equations in $\boldsymbol{n}$ adjoint states, we can eliminate the adjoint states $\lambda^{r}=\left[\begin{array}{llll}\lambda_{1} & \lambda_{2} & \ldots & \lambda_{n}\end{array}\right]$ to obtain expression (20).

Theorem 3: For a system of the form of eq. (2) and a performance index of the form of eq. (1), the singular trajectories can be tracked by dymamic state feedback when $\mathrm{s} \leqslant(\mathrm{n}-3)$. The optimal state feedback law is the solution of an $(\mathrm{n}-\mathrm{s}-2)$ th-order ordinary differential equation of the form

$$
\Phi\left(x, u, \frac{\mathrm{d} u}{\mathrm{~d} t}, \ldots, \frac{\mathrm{d}^{n-s-2} u}{\mathrm{~d} t^{n-s-2}}\right)=0
$$

where $\Phi$ is an algebraic function. This is obtained by setting the determinant of

$g(x)$

$\operatorname{ad}_{f}^{1} g(x)$

$a d_{f}^{s} g(x)$

$a d_{f}^{s+1} g(x)+\left[g, a d_{f}^{s} g\right](x) u(t)$

$a d_{f}^{s+2} g(x)+\left[g, a d_{f}^{s+1} g\right](x) u(t)$

$+\left(I \frac{\mathrm{d}}{\mathrm{d} t}-\frac{\partial f(x)}{\partial x}-\frac{\partial g(x)}{\partial x} u(t)\right)\left(\left[g, a d_{f}^{s} g\right](x) u(t)\right)$

$a d_{f}^{n-1} g(x)+\sum_{k=0}^{n-s-2}\left(I \frac{\mathrm{d}}{\mathrm{d} t}-\frac{\partial f(x)}{\partial x}-\frac{\partial g(x)}{\partial x} u(t)\right)^{k}$

$\times\left(\left[g, a d_{f}^{n-k-2} g\right](x) u(t)\right)$

equal to zero. In the above equations, I is the identity matrix and $d(\cdot) / d t$ is the total time derivative operator

$$
\frac{\mathrm{d}(\cdot)}{\mathrm{d} t}=(f(x)+g(x) u(t)) \frac{\partial(\cdot)}{\partial x}+\frac{\partial(\cdot)}{\partial t} .
$$

Proof: From proposition 2, the first-order necessary conditions for singular extremal are

$$
\lambda^{T} g(x)=0
$$


obtain (n-s-2) more equations:

$$
\begin{aligned}
& \lambda^{T}\left\{a d_{f}^{s+2} g(x)+\left[g, a d_{f}^{s+1} g\right](x) u(t)\right. \\
& +\left(I \frac{\mathrm{d}}{\mathrm{d} t}-\frac{\partial f(x)}{\partial x}-\frac{\partial g(x)}{\partial x} u(t)\right) \\
& \left.\times\left(\left[g, \operatorname{ad}_{f}^{s} g\right](x) u(t)\right)\right\}=0 \\
& \lambda^{T}\left\{a d_{f}^{s+3} g(x)+\left[g, a d_{f}^{s+2}(g)\right](x) u(t)\right. \\
& +\left(I \frac{\mathrm{d}}{\mathrm{d} t}-\frac{\partial f(x)}{\partial x}-\frac{\partial g(x)}{\partial x} u(t)\right) \\
& \left.\times\left(\left[g, a d_{f}^{s+1} g\right](x) u(t)\right)\right\} \\
& +\lambda^{T}\left(I \frac{\mathrm{d}}{\mathrm{d} t}-\frac{\partial f(x)}{\partial x}-\frac{\partial g(x)}{\partial x} u(t)\right)^{2} \\
& \times\left(\left[g, a d_{f}^{s} g\right](x) u(t)\right)=0 \\
& \text { : } \\
& \lambda^{T}\left\{a d_{f}^{n-1} g(x)+\sum_{k=0}^{n-s-2}\left(I \frac{\mathrm{d}}{\mathrm{d} t}-\frac{\partial f(x)}{\partial x}-\frac{\partial g(x)}{\partial x} u(t)\right)^{k}\right. \\
& \left.x\left(\left[g, a d_{f}^{n-k-2} g\right](x) u(t)\right)\right\}=0 .
\end{aligned}
$$

Equations (22) and (23) constitute a set of $n$ equations in $n$ adjoint variables which can be eliminated to give the dynamic state feedback law. This is obtained by setting the determinant of the vector fields in eq. (21) equal to zero.

\section{END-POINT OPTIMIZATION FOR TIME-VARYING SYSTEMS}

In the previous section, we derived state feedback laws for end-point optimization for processes which are described by time-invariant models. These feedback laws can be used directly for a large class of yield optimization problems in batch chemical reactors. However, there is a class of yield optimization problems which fall in the framework of end-point optimization formulated in this paper with the exception that the process models have time appear explicitly as a parameter or have parameters that vary with time. For example, in biological processes, the system models can have time-varying parameters to account for intracellular events. For instance, Miura et al. (1975) developed a mathematical model to describe growth of a microorganism; in this model, time appears explicitly as a parameter. Staniskis and Levisauskas (1984) developed a mathematical model for the production of $\alpha$-amylase by $B$. subtilis; this model contains time-varying parameters. End-point optimization of such processes is important in terms of maximizing production of biomass or product yield and this motivates the extension of the results de- veloped in the previous section to time-varying systems.

Consider the following optimization problem.

Minimize the performance index

$$
J=\phi\left(x\left(t_{f}\right)\right)
$$

subject to the dynamics

$$
\begin{aligned}
\dot{x} & =f(x, t)+g(x, t) u \quad 0 \leqslant t \leqslant t_{f} \\
x(0) & =x_{0}
\end{aligned}
$$

where $u$ is the (scalar) manipulated input, $x$ is the $n$-vector of states, $t_{f}$ is the final time, $\phi$ is a nonconstant scalar function of the system states and $f$ and $g$ are vector functions that depend explicitly on the time variable, $t$.

The classical optimal control perspective presented in the previous section is applicable to the above system. Thus, in the singular region, the first-order necessary conditions are given by eqs (6) and (7), where $g(x)$ is replaced by $g(x, t)$. However, the recursive "ad" notation has to be modified to take into account the explicit dependence of the system model on time.

We define modified Lie brackets of the time-varying system (25) as

$$
\begin{aligned}
& \overline{a d}_{f}^{o} g(x, t)=g(x, t) \\
& \overline{a d}_{f}^{1} g(x, t)=[f, g](x, t)+\frac{\partial g(x, t)}{\partial t} \\
& \overline{a d}_{f}^{2} g(x, t)=\left[f, \overline{a d}_{f}^{1} g\right](x, t)+\frac{\partial \overline{a d}_{f}^{1} g(x, t)}{\partial t}
\end{aligned}
$$

$$
\overline{a d}_{f}^{k} g(x, t)=\left[f, \overline{a d}_{f}^{k-1} g\right](x, t)+\frac{\partial \overline{a d}_{f}^{k-1} g(x, t)}{\partial t} .
$$

As in the case of time-invariant systems, we make the standing assumption that the vector fields $g(x, t)$, $\overline{a d}_{f}^{1} g(x, t), \ldots, \overline{a d}_{f}^{n-1} g(x, t)$ are linearly independent almost everywhere in $x$ and $t$. This assumption is equivalent to

$$
\operatorname{det}\left[g(x, t) \quad \overline{a d}_{f}^{1} g(x, t) \quad \ldots \quad \overline{a d}_{f}^{n-1} g(x, t)\right] \not \equiv 0 .
$$

Definition 2: The system governed by eq. (25) has a finite degree of singularity $\mathrm{s}$ if

$$
\begin{aligned}
{\left[g, \overline{a d}_{f}^{v} g\right](x, t) \in \operatorname{span} } & \left\{g(x, t), \overline{a d}_{f}^{1} g(x, t), \ldots\right. \\
& \left.\overline{a d}_{f}^{v} g(x, t)\right\}, \text { for all } v \leqslant s-1
\end{aligned}
$$

$\left[g, \overline{a d}_{f}^{s} g\right](x, t) \notin \operatorname{span}\left\{g(x, t), \overline{a d}_{f}^{1} g(x, t), \ldots\right.$

$$
\left.\overline{a d}_{f}^{s} g(x, t)\right\} \text {. }
$$

The system governed by eq. (25) has an infinite degree of singularity $(s=\infty)$ if

$$
\begin{array}{r}
{\left[g, \overline{a d}_{f}^{v} g\right](x, t) \in \operatorname{span}\left\{g(x, t), \overline{a d}_{f}^{1} g(x, t), \ldots,\right.} \\
\left.\qquad \overline{a d}_{f}^{v} g(x, t)\right\}, \text { for all } v \geqslant 0 .
\end{array}
$$


It follows immediately from the definition that $1 \leqslant s \leqslant(n-2)$ or $s=\infty$.

Using the definitions of the modified Lie brackets of the system and the degree of singularity, the firstorder necessary conditions on the singular region can be written as

$$
\begin{aligned}
& \lambda^{T} g(x, t)=0 \\
& \lambda^{T} \overline{a d}_{f}^{1} g(x, t)=0 \\
& \lambda^{T} \overline{a d}_{f}^{2} g(x, t)=0 \\
& \vdots \\
& \lambda^{T} \overline{a d}_{f}^{s} g(x, t)=0 \\
& \lambda^{T} \overline{a d}_{f}^{s+1} g(x, t)+\lambda^{T}\left[g, \overline{a d}_{f}^{s} g\right](x, t) u=0 .
\end{aligned}
$$

Furthermore, the second-order necessary condition for optimality takes the form

$$
(-1)^{(s+1) / 2} \lambda^{r}\left[g, \overline{a d}_{f}^{s} g\right](x, t) \geqslant 0 .
$$

The following theorems generalize the results of the previous section to tîme-varying systems. Theorem 4 refers to the case $s=\infty$ and theorem 5 to the case of finite $s$.

Theorem 4: Consider a system of the form of eq. (25) with degree of singularity $\mathrm{s}=\infty$ and a performance index of the form of eq. (24).

(i) If $\mathrm{g}(\mathrm{x}, \mathrm{t}), \overline{\mathrm{ad}}_{\mathrm{f}}^{1} \mathrm{~g}(\mathrm{x}, \mathrm{t}), \ldots, \overline{\mathrm{ad}}_{\mathrm{p}}^{\mathrm{n}-1} \mathrm{~g}(\mathrm{x}, \mathrm{t})$ are linearly independent for all $x$ and $t$, then a singular extremal does not exist.

(ii) If $\mathrm{g}(\mathrm{x}, \mathrm{t}), \overline{\mathrm{ad}}_{\mathrm{f}}^{1} \mathrm{~g}(\mathrm{x}, \mathrm{t}), \ldots, \overline{\mathrm{ad}}_{\mathrm{f}}^{\mathrm{n}-1} \mathrm{~g}(\mathrm{x}, \mathrm{t})$ are linearly independent almost everywhere, then the state trajectories corresponding to a singular extremal satisfy the following relationship:

$\bar{S}(x, t) \equiv \operatorname{det}\left[g(x, t) \overline{a d}_{f}^{1} g(x, t) \ldots \overline{a d}_{f}^{n-1} g(x, t)\right]=0$.

The first $n$ equations can be written in compact form as

$$
\lambda^{T}\left[g(x, t) \quad \overline{a d}_{f}^{1} g(x, t) \quad \ldots, \overline{a d}_{f}^{n-1} g(x, t)\right]=0 .
$$

Since $\lambda(t)$ is an absolutely continuous nonzero vector function in $\mathbb{R}^{n}$, it follows that

$$
\operatorname{det}\left[g(x, t) \quad \overline{a d}_{f}^{1} g(x, t) \quad \ldots \quad \overline{a d}_{f}^{n-1} g(x, t)\right]=0 .
$$

for all $x$ and $t$ in some nonempty set in $\mathbb{R}^{n} \times \mathbb{R}$. This contradicts the assumption of global linear independence of $g(x, t), \overline{a d}_{f}^{1} g(x, t), \ldots, \overline{a d}_{f}^{n-1} g(x, t)$.

Proof of (ii): The first-order necessary conditions on the singular extremal are given by eq. (33). Using exactly the same argument as in the proof of (i), we conclude that the state trajectories corresponding to the singular control must satisfy the following relationship:

$$
\bar{s}(x, t)=0 .
$$

It can be easily shown on the lines of corollary 1 that controllable linear time-varying systems do not possess singular extremals. Furthermore, it is observed from theorem 4 that optimality may correspond to loss of state controllability. Whenever a singular extremal exists for a system with infinite degree of singularity, the problem of synthesizing a state feedback to track the singular trajectories reduces to a standard regulation problem for the time-varying output, i.e.

$$
y=\bar{S}(x, t)
$$

with zero set point.

Theorem 5: Consider a system of the form of eq. (25) and a performance index of the form of eq. (24).

(i) For $\mathrm{s}=(\mathrm{n}-2)$, the singular trajectories can be tracked by the following state feedback:

$$
u=-\frac{\operatorname{det}\left[\begin{array}{lllll}
g(x, t) & \overline{a d}_{f}^{1} g(x, t) & \ldots & \overline{a d}_{f}^{n-1} g(x, t)
\end{array}\right]}{\operatorname{det}\left[\begin{array}{lllll}
g(x, t) & \overline{a d}_{f}^{1} g(x, t) & \ldots & \overline{a d}_{f}^{n-2} g(x, t) & {\left[g, \overline{a d}_{f}^{n-2} g\right](x, t)}
\end{array}\right]}
$$

Proof of (i): We prove this part of the theorem by contradiction. Let us assume that a singular extremal exists for the system (25) with degree of singularity $s=\infty$. The first-order necessary conditions on the singular extremal are as follows:

$$
\begin{aligned}
\lambda^{T} g(x, t) & =0 \\
\lambda^{T} \overline{a d}_{j}^{1} g(x, t) & =0 \\
\lambda^{T} \overline{a d}_{f}^{2} g(x, t) & =0 \\
\vdots & \\
\lambda^{T} \overline{a d}_{f}^{n-1} g(x, t) & =0
\end{aligned}
$$

(ii) For $\mathrm{s} \leqslant(\mathrm{n}-3)$, the singular trajectories can be tracked by a dynamic state feedback which is calculated by setting the determinant of the following vector fields equal to zero.

$$
\begin{aligned}
& g(x, t) \\
& \overline{a d}_{f}^{1} g(x, t) \\
& \quad \vdots \\
& \overline{a d}_{f}^{s} g(x, t) \\
& \overline{a d}_{f}^{s+1} g(x, t)+\left[g, \overline{a d}_{f}^{s} g\right](x, t) u(t) \\
& \overline{a d}_{f}^{s+2} g(x, t)+\left[g, \overline{a d}_{f}^{s+1} g\right](x, t) u(t)
\end{aligned}
$$




$$
\begin{aligned}
& +\left(I \frac{\mathrm{d}}{\mathrm{d} t}-\frac{\partial f(x, t)}{\partial x}-\frac{\partial g(x, t)}{\partial x} u(t)\right) \\
& \times\left(\left[g, \overline{a d}_{S}^{s} g\right](x, t) u(t)\right) \\
& \quad \vdots \\
& \overline{a d_{f}^{n}-1} g(x, t)+\sum_{k=0}^{n-s-2}\left(I \frac{\mathrm{d}}{\mathrm{d} t}-\frac{\partial f(x, t)}{\partial x}-\frac{\partial g(x, t)}{\partial x} u(t)\right)^{k} \\
& \times\left(\left[g, \overline{a d}_{f}^{n-k-2} g\right](x, t) u(t)\right) .
\end{aligned}
$$

Proof of (i): For $s=n-2$, the first-order necessary conditions can be written as

$$
\begin{gathered}
\lambda^{T} g(x, t)=0 \\
\lambda^{T} \overline{a d}_{f}^{1} g(x, t)=0 \\
\lambda^{T} \overline{a d}_{f}^{2} g(x, t)=0 \\
\vdots \\
\lambda^{T} \overline{a d}_{f}^{n-2} g(x, t)=0 \\
\lambda^{T} \overline{a d}_{f}^{n-1} g(x, t)+\lambda^{T}\left[g, \overline{a d}_{f}^{n-2} g\right](x, t) u=0 .
\end{gathered}
$$

Since there are $n$ equations in $n$ adjoint states, we can eliminate the adjoint states $\lambda^{T}=\left[\lambda_{1}, \lambda_{2}, \ldots, \lambda_{n}\right]$ to obtain expression (36).

Proof of (ii): For $s \leqslant(n-3)$, the first-order necessary conditions can be written as eq. (31) which constitute $(s+2)$ equations in $n$ adjoint states. Differentiating the last equation in eq. (31) $(n-s-2)$ times we obtain $(n-s-2)$ more equations, i.e.

$$
\begin{aligned}
& \lambda^{r}\left\{\overline{a d}_{f}^{s+2} g(x, t)+\left[g, \overline{a d}_{f}^{s+1} g\right](x, t) u(t)\right. \\
& \quad+\left(I \frac{\mathrm{d}}{\mathrm{d} t}-\frac{\partial f(x, t)}{\partial x}-\frac{\partial g(x, t)}{\partial x} u(t)\right) \\
& \left.\quad \times\left(\left[g, \overline{a d}_{f}^{s} g\right](x, t) u(t)\right)\right\}=0 \\
& \lambda^{r}\left\{\overline{a d}_{f}^{s+3} g(x, t)+\left[g, \overline{a d_{f}^{s+2}} g\right](x, t) u(t)\right. \\
& \quad+\left(I \frac{\mathrm{d}}{\mathrm{d} t}-\frac{\partial f(x, t)}{\partial x}-\frac{\partial g(x, t)}{\partial x} u(t)\right) \\
& \left.\quad \times\left(\left[g, \overline{a d}_{f}^{s+1} g\right](x, t) u(t)\right)\right\} \\
& \quad+\lambda^{r}\left(I \frac{\mathrm{d}}{\mathrm{d} t}-\frac{\partial f(x, t)}{\partial x}-\frac{\partial g(x, t)}{\partial x} u(t)\right)^{2} \\
& \quad \times\left(\left[g, \overline{a d}_{f}^{s} g\right](x, t) u(t)\right)=0 \\
& \quad \vdots \\
& \lambda^{r}\left\{\overline{a d}_{f}^{n-1} g(x, t)\right. \\
& \quad+\sum_{k=0}^{n-s-2}\left(I \frac{\mathrm{d}}{\mathrm{d} t}-\frac{\partial f(x, t)}{\partial x}-\frac{\partial g(x, t)}{\partial x} u(t)\right)^{k} \\
& \quad \times\left(\left[g, \overline{a d}{ }_{f}^{n-k-2} g\right](x, t) u(t)\right\}=0 .
\end{aligned}
$$

Equations (31) and (39) constitute a set of $n$ equations in $n$ adjoint variables which can be eliminated to give the dynamic state feedback law. This is obtained by setting the determinant of the vector fields in eq. (37) equal to zero.

If the time dependence of the system model is in terms of time-varying parameters, $f(x, k(t))$ and $g(x, k(t))$, where $k(t)$ is the vector of time-varying parameters, it is necessary to express the quantities $\overline{a d}_{f}^{1} g, \overline{a d}_{f}^{2} g, \ldots$ in terms of the partial derivatives of $f$ and $g$ with respect to $k$ and the time derivatives $\mathrm{d} k / \mathrm{d} t, \mathrm{~d}^{2} k / \mathrm{d} t^{2}, \ldots$ to obtain concrete formulae for the state feedback law. This can be done in a very straightforward manner:

$$
\begin{aligned}
\overline{a d}_{f}^{1} g(x, t) & =[f, g](x, t)+\frac{\partial g(x, t)}{\partial t} \\
& =a d_{f}^{1} g(x, t)+\frac{\partial g(x, t)}{\partial k} \frac{\mathrm{d} k}{\mathrm{~d} t} \\
\overline{a d}_{f}^{2} g(x, t) & =\left[f, \overline{a d}_{f}^{1} g\right](x, t)+\frac{\partial \overline{a d}_{f}^{1} g(x, t)}{\partial t} \\
& =a d_{f}^{2} g(x, t)+\left(\left[f, \frac{\partial g}{\partial k}\right](x, t)\right. \\
& \left.+\frac{\partial a d_{f}^{1} g(x, t)}{\partial k}+\frac{\partial^{2} g(x, t)}{\partial k^{2}} \frac{\mathrm{d} k}{\mathrm{~d} t}\right) \frac{\mathrm{d} k}{\mathrm{~d} t} \\
& +\frac{\partial g(x, t)}{\partial k} \frac{\mathrm{d}^{2} k}{\mathrm{~d} t^{2}} \\
& \vdots
\end{aligned}
$$

In general, $\overline{a d}_{f}^{J} g(x, t)$ will be expressed as a function of $x, k(t), \mathrm{d} k / \mathrm{d} t, \ldots, \mathrm{d}^{j} k / \mathrm{d} t^{j}$.

\section{IMPLEMENTATION}

The results obtained in the previous sections provide optimal feedback laws strictly in the singular region. In the nonsingular region, the manipulated input takes the minimum or the maximum value. To determine what values (minimum or maximum) the manipulated input takes in the nonsingular region as well as the switching times between the singular and nonsingular regions, one has to integrate, off-line, the adjoint equations in addition to the state equations. The singular region corresponds to vanishing of the switching function $\lambda^{T} g$, whereas the nonsingular regions correspond to nonzero values of the switching function. To determine whether the singular control is indeed optimal, one has to check the higher-order necessary conditions as well.

To implement the optimal state feedhack laws as a closed-loop scheme, the switching times between singular and nonsingular regions are required. These switching times can be calculated analytically only for a restricted class of models. In most practical situations, these have to be computed numerically. From the off-line solution of the first-order necessary conditions of optimality, the switching times and the values of the system states at the junctions between singular 
and nonsingular regions are known. This information can be used to set up on-line criteria for the onset of the singular region in terms of the system states. For instance, Modek (1988) determined the onset of the singular interval in ethanol fermentation by using an expression relating cell mass and substrate concentrations at the junction of the nonsingular and singular regions. Alternatively, one can use the a priori calculated switching times determined from the offline analysis. In this case a successful feedback implementation of the optimal feedback law depends on how sensitive the final performance index is to errors in the switching times. In example 3 , it will be seen that the final performance index is not very sensitive to small errors in the switching time. When $s \leqslant(n-3)$, we also need initial conditions for the dynamic state feedback law. These initial conditions have to be set up in a similar fashion as the switching times.

If the model parameters are known a priori, the feedback laws derived in the previous sections can be used in conjunction with state estimators. Such an implementation can attenuate errors in the initial conditions and process disturbances. If some of the model parameters are not accurately known a priori, these need to be estimated on-line. In this situation, a parameter estimation algorithm is also required. If the model parameters are time-varying and the functional form of these time-varying parameters is not known, the theory developed in the preceding sections can be used as a basis for an adaptive optimal state feedback. In this approach, the optimal state feedback is coupled with a state and parameter estimation algorithm. The state and parameter estimation algorithm provides estimates of the system states and model parameters which are used to update the state feedback law periodically.

A variety of stochastic methods such as extended Kalman filtering (Jazwinski, 1970), and predictionerror identification (Ljung, 1985) as well as deterministic methods such as nonlinear least squares, have been proposed in the literature to estimate the system states and parameters. These methods have been applied to a variety of batch systems for state and parameter estimation. For instance, Schuler and Papadopoulou (1986) developed a decoupled nonlinear estimator based on an extended Kalman filter for the real-time estimation of the chain-length distribution and conversions in a batch polystyrene reactor. Ellis et al. (1988) applied a two time-scale filtering technique to estimate the temperature, monomer conversion, initiator conversion, and the entire molecular weight distribution in a methyl methacrylate batch polymerization. Stephanopoulos and San (1984) demonstrated the use of Kalman filtering to estimate the specific growth rate and cell mass in a batch fermentation using on-line off-gas data. Numerical implementation aspects relating to coupling of a state and parameter estimation algorithm with the optimal feedback laws developed in this work will be addressed in a future communication.

\section{ILLESTRATIVE EXAMPI.FS}

In this section we will illustrate, through four simulation examples, applications for the theory developed in the preceding sections.

\section{Example 1: linear system}

We consider a yield optimization problem in a semi-batch chemical reactor where the following reactions are occurring:

$$
\begin{aligned}
& A \rightarrow B \\
& B \rightarrow C \\
& A \rightarrow D
\end{aligned}
$$

The species $A$ is being fed to the reactor. All reactions follow first-order kinetics. The unsteady-state mass balance equations are given by

$$
\begin{aligned}
\frac{\mathrm{d}\left(C_{A} V\right)}{\mathrm{d} t} & =-k_{B} C_{A} V-k_{D} C_{A} V+s_{F} u \\
\frac{\mathrm{d}\left(C_{B} V\right)}{\mathrm{d} t} & =k_{B} C_{A} V-k_{C} C_{B} V \\
\frac{\mathrm{d}\left(C_{D} V\right)}{\mathrm{d} t} & =k_{D} C_{A} V \\
\frac{\mathrm{d} V}{\mathrm{~d} t} & =u
\end{aligned}
$$

where the manipulated input, $u$, is the feed rate of species $A$. The objective is to operate the batch reactor so that the total amount of $B$ is maximized at a prespecified final time $t_{f}$. Thus, the performance index to minimize is

$$
J=-\left.C_{B} V\right|_{t=i s}
$$

Define

$$
\begin{aligned}
& x_{1}=C_{A} V \\
& x_{2}=C_{B} V \\
& x_{3}=C_{D} V \\
& x_{4}=V
\end{aligned}
$$

The optimization problem can be stated as follows. Minimize

$$
J=x_{2}\left(t_{f}\right)
$$

subject to

$\frac{\mathrm{d}}{\mathrm{d} t}\left[\begin{array}{c}x_{1} \\ x_{2} \\ x_{3} \\ x_{4}\end{array}\right]=\left[\begin{array}{c}-k_{B} x_{1}-k_{D} x_{1} \\ k_{B} x_{1}-k_{C} x_{2} \\ k_{D} x_{1} \\ 0\end{array}\right]+\left[\begin{array}{c}s_{F} \\ 0 \\ 0 \\ 1\end{array}\right] u$

The system model is of the form

$$
\dot{x}=f(x)+g(x) u
$$


144

where

$$
\begin{aligned}
& x=\left[\begin{array}{l}
x_{1} \\
x_{2} \\
x_{3} \\
x_{4}
\end{array}\right] \\
& f(x)=\left[\begin{array}{c}
-k_{B} x_{1}-k_{D} x_{1} \\
k_{B} x_{1}-k_{C} x_{2} \\
k_{D} x_{1} \\
0
\end{array}\right] \\
& g(x)=\left[\begin{array}{c}
s_{F} \\
0 \\
0 \\
1
\end{array}\right]
\end{aligned}
$$

It can be easily verified that the vector fields $g(x)$, $a d_{f}^{1} g(x), a d_{f}^{2} g(x), a d_{f}^{3} g(x)$ are linearly independent constant vectors and so the system is controllable everywhere. We note from the vector fields $f(x)$ and $g(x)$ that the system under consideration is linear. Thus, this system does not possess a singular extremal

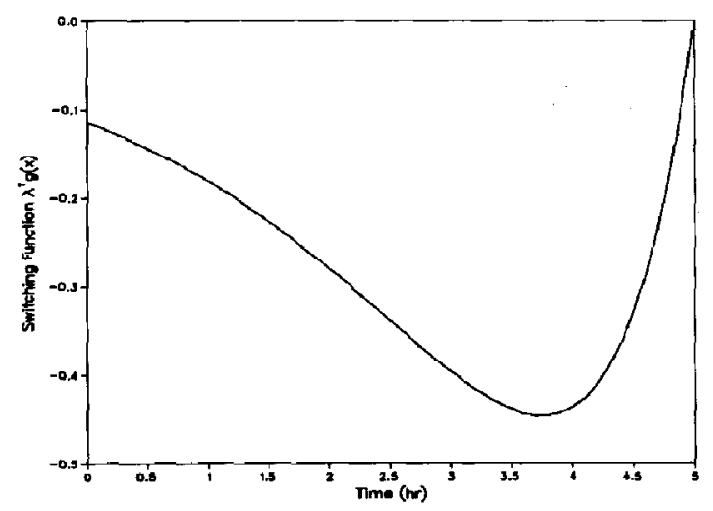

Fig. 1. Profile of switching function $\lambda^{T} g(x)$ (example 1).

Table 1(a). Model parameters

\begin{tabular}{ll}
\hline$k_{B}$ & $1.00 \mathrm{l} / \mathrm{h}$ \\
$k_{C}$ & $0.501 / \mathrm{h}$ \\
$k_{D}$ & $0.201 / \mathrm{h}$ \\
$s_{F}$ & $10.00 \mathrm{~kg} / \mathrm{m}^{3}$ \\
$u_{\max }$ & $5.00 \times 10^{-3} \mathrm{~m}^{3} / \mathrm{h}$ \\
$t_{r}$ & $5.00 \mathrm{~h}$ \\
\hline
\end{tabular}

Table 1(b). Initial conditions

\begin{tabular}{ll}
\hline$C_{A}(0)$ & $1.0 \mathrm{~kg} / \mathrm{m}^{3}$ \\
$C_{B}(0)$ & $0.0 \mathrm{~kg} / \mathrm{m}^{3}$ \\
$C_{D}(0)$ & $0.0 \mathrm{~kg} / \mathrm{m}^{3}$ \\
$V(0)$ & $1.0 \times 10^{-3} \mathrm{~m}^{3}$ \\
\hline
\end{tabular}

(corollary 1), and therefore the optimal control is bang-bang in nature. The sign of $\lambda^{T} g(x)=s_{F} \lambda_{1}(t)$ determines if the maximum value or the minimum value of $u$ should be used. It can be easily seen that

$$
\begin{aligned}
\lambda_{1}(t)= & \frac{k_{B}}{k_{C}-k_{\mathrm{B}}-k_{D}}\left\{\exp \left[k_{C}\left(t-t_{f}\right)\right]\right. \\
& \left.-\exp \left[\left(k_{B}+k_{D}\right)\left(t-t_{f}\right)\right]\right\} .
\end{aligned}
$$

Figure 1 shows the time profile of $\lambda^{T} g(x)$ for the model parameters given in Table $1(a)$ and the initial conditions given in Table $1(b)$. Since $\lambda^{T} g(x)$ is always negative in this region, the optimal operation is to use the maximum feed rate $u_{\max }$ throughout the batch time. Figure 2 shows the time profile of species $B$ when the maximum feed rate is used.

\section{Example 2: $s=\infty$}

We consider a fed-batch fermentation for the production of baker's yeast (Menawat et al., 1987). The unsteady-state material balances are as follows (see Notation for explanation of symbols):

$$
\begin{aligned}
\frac{\mathrm{d}(X V)}{\mathrm{d} t} & =\mu(S, t) X V \\
\frac{\mathrm{d}(S V)}{\mathrm{d} t} & =-\frac{\mu(S, t) X V}{Y}+s_{F} u \\
\frac{\mathrm{d} V}{\mathrm{~d} t} & =u .
\end{aligned}
$$

The objective is to maximize the cell mass at a prespecified final time, $t_{\boldsymbol{f}}$. Thus

$$
J=-\left.X V\right|_{t=t_{f}}
$$

The manipulated variable, $u$, is the feed rate of the substrate. The specific growth rate, $\mu$, is given by the Haldane-Monod function (Edwards, 1970)

$$
\mu(S, t)=\frac{\mu_{m}(t) S}{K_{1}+S} \frac{K_{2}}{K_{2}+S} .
$$

The parameter $\mu_{m}(t)$ decreases with time due to degradation of intracellular enzymes and its time variation

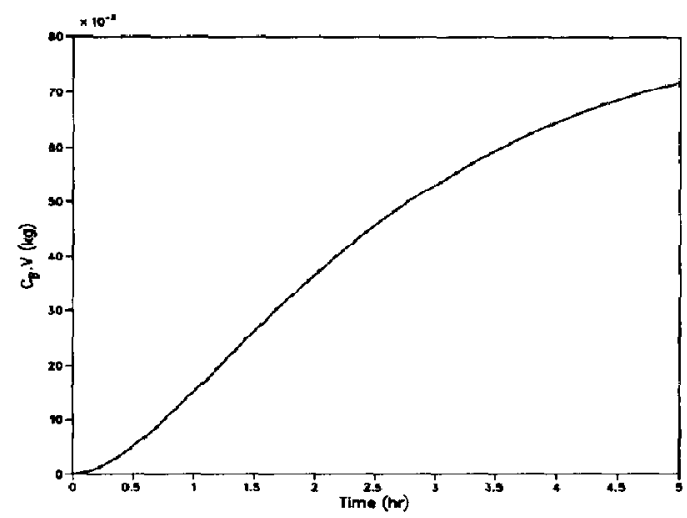

Fig. 2, Optimal time profile of species B (example 1). 
is shown in Fig. 3. Condition (27) is violated every" where for this system. In this situation, we need to do model reduction to eliminate the redundant state equations, so that condition (27) is satisfied almost everywhere in $x$. It can be easily shown that $X, S$ and $V$ are algebraically related by

$$
\begin{aligned}
\left(\frac{X V}{Y}+S V\right)-\left(\frac{X(0) V(0)}{Y}+\right. & S(0) V(0)) \\
& =s_{F}(V-V(0)) .
\end{aligned}
$$

Thus, there are only two independent equations. Defining

$$
\begin{aligned}
& x_{1}=X V \\
& x_{2}=V
\end{aligned}
$$

and eliminating $S$ from eq. (50)

$$
\begin{aligned}
S= & s_{F}-\frac{1}{Y}\left(\frac{x_{1}-X(0) V(0)}{x_{2}}\right) \\
& +\left(\frac{S(0) V(0)-s_{F} V(0)}{x_{2}}\right)
\end{aligned}
$$

the model can be put in the form

$$
\frac{\mathrm{d}}{\mathrm{d} t}\left[\begin{array}{l}
x_{1} \\
x_{2}
\end{array}\right]=\left[\begin{array}{c}
\hat{\mu}\left(x_{1}, x_{2}, t\right) x_{1} \\
0
\end{array}\right]+\left[\begin{array}{l}
0 \\
1
\end{array}\right] u
$$

where $\hat{\mu}$ is obtained by substituting $S$ from eq. (52) into the expression for $\mu$ [eq. (49)]. For this system

$$
\begin{array}{r}
x=\left[\begin{array}{l}
x_{1} \\
x_{2}
\end{array}\right], \quad f(x, t)=\left[\begin{array}{c}
\hat{\mu}\left(x_{1}, x_{2}, t\right) x_{1} \\
0
\end{array}\right], \\
g(x, t)=\left[\begin{array}{l}
0 \\
1
\end{array}\right] .
\end{array}
$$

Since $n=2$, we necessarily have $s=\infty$. It can be easily seen that the necessary condition

$$
\operatorname{det}\left[g(x, t) \quad \overline{a d} \frac{1}{s} g(x, t)\right]=0
$$

leads to

$$
\frac{\partial \hat{\mu}}{\partial x_{2}} x_{1}=0
$$

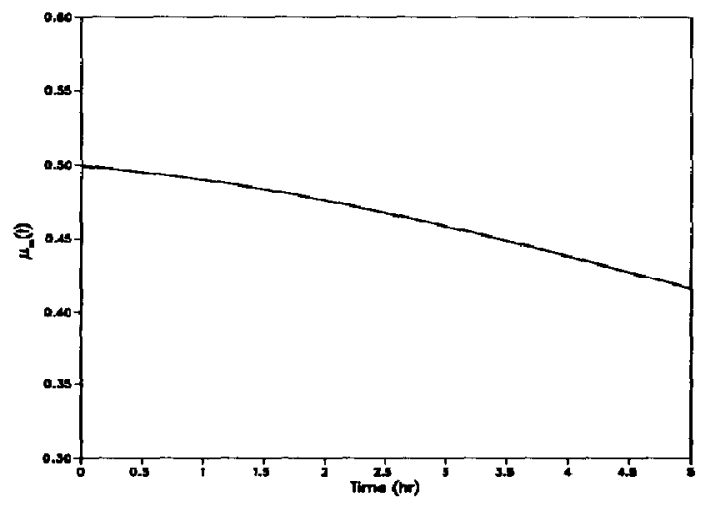

Fig. 3. Profile of time-varying parameter $\mu_{m}(t)$ (example 2).
In terms of the original variables ( $S$ and $X$ ), the above equation takes the form

$$
\frac{\partial \mu}{\partial S}\left(s_{F}-S\right) X=0 .
$$

Since $S \neq s_{F}, X \neq 0$, this further simplifies to

$$
\frac{\partial \mu}{\partial S}=0
$$

which from the kinetic expression (49) for $\mu$ leads to

$$
S-\sqrt{K_{1} K_{2}}=0 .
$$

Thus, the problem of maximizing cell mass at the end of the batch time reduces to regulating the substrate concentration to $\sqrt{K_{1} K_{2}}$. On intuitive grounds also, this solution makes sense. To maximize biomass, the system must operate at that level of $S$ that maximizes the specific growth rate $\mu$.

We use the following state feedback law to regulate the substrate concentration to $\sqrt{K_{1} K_{2}}$ :

$$
u=\frac{(\mu(S, t) X / Y)+\left(\sqrt{K_{1} K_{2}}-S\right)}{\left(s_{F}-S\right) / V} .
$$

Since the reactor starts with a high substrate concentration, there will be an initial nonsingular phase where no substrate is fed. The switch to the singular phase occurs when $S$ reaches the value $\sqrt{K_{1} K_{2}}$. Figures 4 and 5 show the optimal feeding profile, the optimal cell mass profile and the optimal substrate concentration profile for the system parameters in Table 2(a) and initial conditions designated case $A$ in Table 2(b). The first-order necessary condition for optimality $\left(\lambda^{r} g(x)=0\right)$ was verified by integrating the adjoint equations backwards in time and is shown in Fig. 6.

The robustness of the control law to errors in the kinetic parameters was tested. In the control law, the values of parameters $K_{1}$ and $K_{2}$ used were $10 \%$ lower than those of the system parameters shown in Table 2(a). The results are summarized in Table 2(c). It is observed that the performance index is very close to

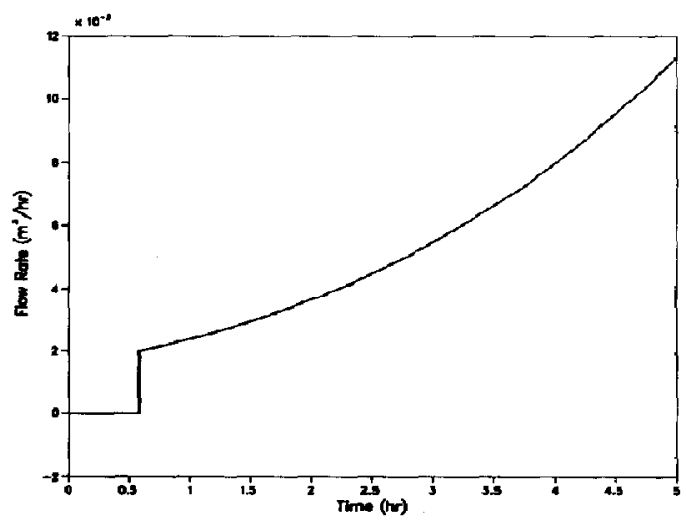

Fig. 4. Optimal input profile (example 2). 


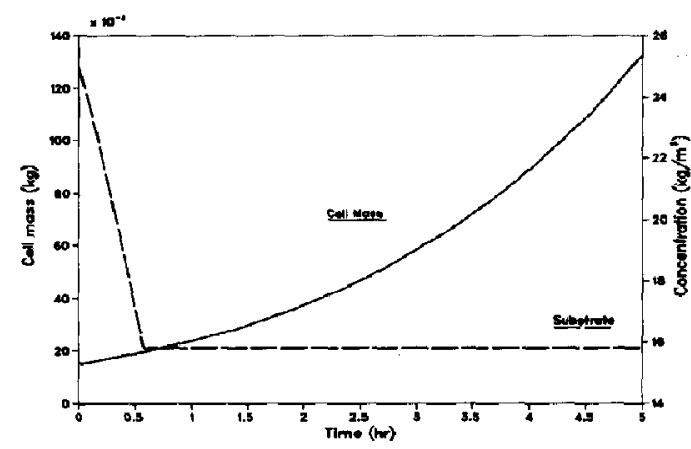

Fig. 5. Optimal cell mass and substrate profiles (example 2): $(-)$ cell mass $(\mathrm{kg}) ;(-\longrightarrow)$ substrate concentration $\left(\mathrm{kg} / \mathbf{m}^{3}\right)$

that obtained in the nominal case, where the true values of the parameters were used in the control law.

The sensitivity of the performance of the control law with respect to errors in the initial conditions is also studied. Feedback implementation was compared to the conventional open-loop implementation. The input profile obtained for the initial conditions designated case $A$ in Table 2(b) was implemented in open loop to the system with initial conditions designated case $B$ in Table 2(b). This was compared to the

Table 2(a). Model parameters

\begin{tabular}{lr}
\hline$K_{1}$ & $0.5 \mathrm{~kg} / \mathrm{m}^{3}$ \\
$K_{2}$ & $500.0 \mathrm{~kg} / \mathrm{m}^{3}$ \\
$s_{F}$ & $25.0 \mathrm{~kg} / \mathrm{m}^{3}$ \\
$Y$ & $0.5 \mathrm{~kg} / \mathrm{kg}$ \\
$t_{f}$ & $5.0 \mathrm{~h}$ \\
\hline
\end{tabular}

Table 2(b). Initial conditions

\begin{tabular}{lc}
\hline Case A \\
\hline$X(0)$ & $15.0 \mathrm{~kg} / \mathrm{m}^{3}$ \\
$S(0)$ & $25.0 \mathrm{~kg} / \mathrm{m}^{3}$ \\
$V(0)$ & $1.0 \times 10^{-3} \mathrm{~m}^{3}$
\end{tabular}

Case $B$

\begin{tabular}{lc}
\hline$X(0)$ & $25.0 \mathrm{~kg} / \mathrm{m}^{3}$ \\
$S(0)$ & $17.5 \mathrm{~kg} / \mathrm{m}^{3}$ \\
$V(0)$ & $1.0 \times 10^{-3} \mathrm{~m}^{3}$ \\
\hline
\end{tabular}

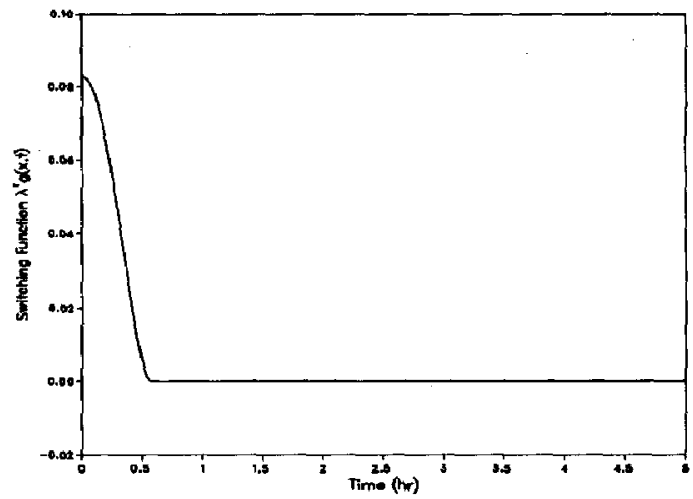

Fig. 6. Profile of switching function $\lambda^{T} g(x, t)$ (example 2).

case where the input profile was determined by the state feedback in closed loop. The condition $S=\sqrt{K_{1} K_{2}}$ was used to calculate the switching time from the nonsingular to singular region in the closedloop implementation. The closed-loop scheme provides optimal performance since the switching time is calculated on-line and the substrate concentration is kept at the optimum level. The results are summarized in Table 2(d). It is observed that there is a reduction of about $3 \%$ in the performance index of the open-loop scheme over the closed-loop scheme.

\section{Example 3: static state feedback}

In this example we consider a yield optimization problem in ethanol production of $S$. cerevisiae in a fed-batch fermentation. The unsteady-state material balances are as follows (see Notation for explanation of symbols):

$$
\begin{aligned}
\frac{\mathrm{d}(X V)}{\mathrm{d} t} & =\mu(S, P, t) X V \\
\frac{\mathrm{d}(S V)}{\mathrm{d} t} & =-\frac{\mu(S, P, t) X V}{Y}+s_{F} u \\
\frac{\mathrm{d}(P V)}{\mathrm{d} t} & =\varepsilon(S, P, t) X V \\
\frac{\mathrm{d} V}{\mathrm{~d} t} & =u
\end{aligned}
$$

Table 2(c). Robustness analysis

\begin{tabular}{lll}
\hline Parameter in control law & Parameters in process & Performance index \\
\hline $\begin{array}{l}K_{1}=0.5, K_{2}=500 \\
\text { All other parameter } \\
\text { values from Table 2(a) }\end{array}$ & $\begin{array}{l}K_{1}=0.5, K_{2}=500 \\
\text { All other parameter } \\
\text { values from Table 2(a) }\end{array}$ & $-132.993 \times 10^{-3} \mathrm{~kg}$ \\
$K_{1}=0.45, K_{2}=450$ & $\begin{array}{l}K_{1}=0.5, K_{2}=500 \\
\text { All other parameter } \\
\text { vall other parameter } \\
\text { values from Table 2(a) }\end{array}$ & $\begin{array}{l} \\
\text { values from Table 2(a) }\end{array}$ \\
\hline
\end{tabular}


Table 2(d). Open-loop vs closed-loop implementation

Open-loop implementation

Initial conditions: case $B$ in Table $2(b)$

Input profile: optimal solution for case $A$

Performance index at final time: $-214.85 \times 10^{-3} \mathrm{~kg}$

Closed-loop implementation

Initial conditions: case $B$ in Table $2(b)$

Input profile: state feedback law from eq. (60)

Performance index at final time: $-221.84 \times 10^{-3} \mathrm{~kg}$

where the manipulated input, $u$, is the feed rate of the substrate. The objective is to maximize the concentration of the product, ethanol, at a prespecified final time $t_{f}$. Thus,

$$
J=-P\left(t_{s}\right)
$$

The following kinetic expressions for $\mu$ and $\varepsilon$ derived by Aiba et al. (1973) are used.

$$
\begin{gathered}
\mu(S, P, t)=\frac{\mu_{0}(t)}{\left(1+P / K_{P}\right)} \frac{S}{\left(K_{S}+S\right)} \\
\varepsilon(S, P, t)=\frac{v_{0}(t)}{\left(1+P / K_{P}^{*}\right)} \frac{S}{\left(K_{S}^{*}+S\right)} .
\end{gathered}
$$

The parameters $\mu_{0}$ and $v_{0}$ decrease with time due to degradation of intracellular enzymes and their time variation is shown in Fig. 7.

Condition (27) is violated everywhere. As in example 2, we do model reduction to eliminate the redundant state equation. It can be easily shown that $X, S$, and $V$ are algebraically related by

$$
\begin{aligned}
\left(\frac{X V}{Y}+S V\right)-\left(\frac{X(0) V(0)}{Y}+\right. & S(0) V(0)) \\
= & s_{F}(V-V(0)) .
\end{aligned}
$$

Thus, there are only three independent equations. Defining

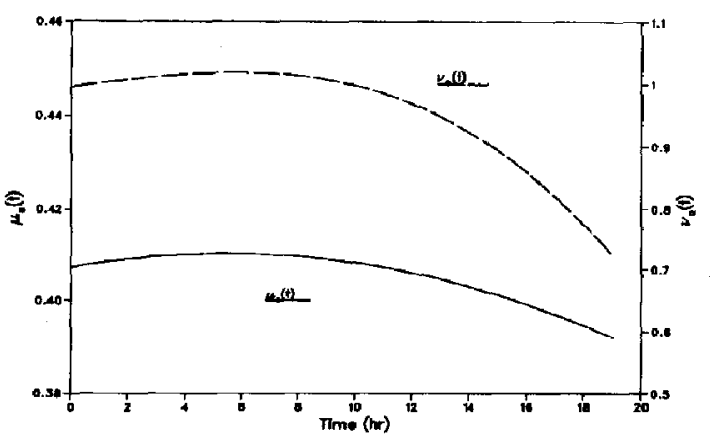

Fig. 7. Profiles of time-varying parameters $\mu_{0}(t)$ and $v_{0}(t)$ (example 3). (- $\mu_{0}(t) ;(-) \nu_{0}(t)$.

the model can be put in the form

$$
\frac{\mathrm{d}}{\mathrm{d} t}\left[\begin{array}{c}
x_{1} \\
x_{2} \\
x_{3}
\end{array}\right]=\left[\begin{array}{c}
\hat{\mu}\left(x_{1}, x_{2}, x_{3}, k(t)\right) x_{1} \\
\hat{\varepsilon}\left(x_{1}, x_{2}, x_{3}, k(t)\right) x_{1} \\
0
\end{array}\right]+\left[\begin{array}{l}
0 \\
0 \\
1
\end{array}\right] u
$$

where $\hat{\mu}$ and $\hat{\varepsilon}$ are obtained by substituting $S$ from eq. (67) and $P=x_{2} / x_{3}$ into the expressions for $\mu$ and $g$. For this system

$$
\begin{array}{r}
x=\left[\begin{array}{l}
x_{1} \\
x_{2} \\
x_{3}
\end{array}\right], f(x, t)=\left[\begin{array}{c}
\hat{\mu}\left(x_{1}, x_{2}, x_{3}, k(t)\right) x_{1} \\
\hat{\varepsilon}\left(x_{1}, x_{2}, x_{3}, k(t)\right) x_{1} \\
0
\end{array}\right] \\
g(x, t)=\left[\begin{array}{l}
0 \\
0 \\
1
\end{array}\right] .
\end{array}
$$

It can easily be verified that now condition (27) is satisfied. Also, $n=3$, and a straightforward calculation shows that $s=1$ and so the state feedback that tracks the singular trajectories will be static. Using expressions (36) and (40) we obtain the following static state feedback law:

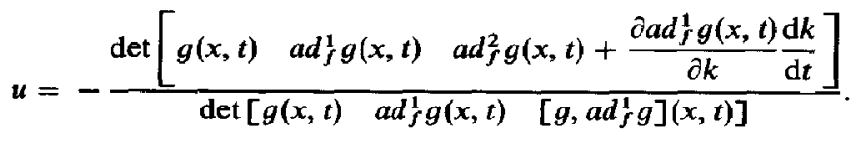

$$
\begin{aligned}
x_{1} & =X V \\
x_{2} & =P V \\
x_{3} & =V \\
k(t) & =\left[\begin{array}{ll}
\mu_{0}(t) & v_{0}(t)
\end{array}\right]^{T}
\end{aligned}
$$

and eliminating the variable $S$ from eq. (65),

$$
\begin{aligned}
S= & s_{F}-\frac{1}{Y}\left(\frac{x_{1}-X(0) V(0)}{x_{3}}\right) \\
& +\left(\frac{S(0) V(0)-s_{F} V(0)}{x_{3}}\right)
\end{aligned}
$$

Since the fermentor starts with a high substrate concentration, there is an initial nonsingular phase where no substrate is fed, followed by a singular phase which involves substrate feeding. In this region there is somc accumulation of substrate. Substrate feeding leads to enhancement of product formation. However, the product also gets diluted due to increase in volume. Since these are competing effects, there will be a short nonsingular phase at the end to use up the accumulated substrate. Thus, on intuitive grounds, we expect to have three phases-nonsingular, singular, nonsingular with two switching times to be calculated. The 
presence of the second nonsingular region is further necessitated by the fact that the terminal conditions for the adjoint states, $\lambda$, are incompatible with the first-order necessary condition on the singular region, $\lambda^{T} g(x, t)=0$.

A two-dimensional search was performed numerically as described in Lim et al. (1986) to get initial estimates of the two switching times. These estimates were further refined till the first-order condition $\lambda^{T} g(x, t)=0$ was satisfied on the singular region. Figures 8 and 9 show the optimal feeding profile and the corresponding product profile for the model parameters in Table 3(a) and the initial conditions designated case $A$ in Table 3(b). Figure 10 depicts the corresponding time profile of the switching function $\left(\lambda^{T} g(x, t)\right)$ and the second-order condition $\left(-\lambda^{T}\left[g, \overline{a d}_{f}^{1} g\right](x, t)\right)$. The switching function vanishes on the singular time interval and is positive everywhere else. The positivity of the switching function is consistent with $u(t)$ taking its minimal value $(u=0)$ in the nonsingular intervals. Furthermore, the secondorder condition $-\lambda^{T}\left[g, \overline{a d}_{f}^{1} g\right](x, t) \geqslant 0$ is satisfied in the singular region and so the singular extremal is indeed optimal.

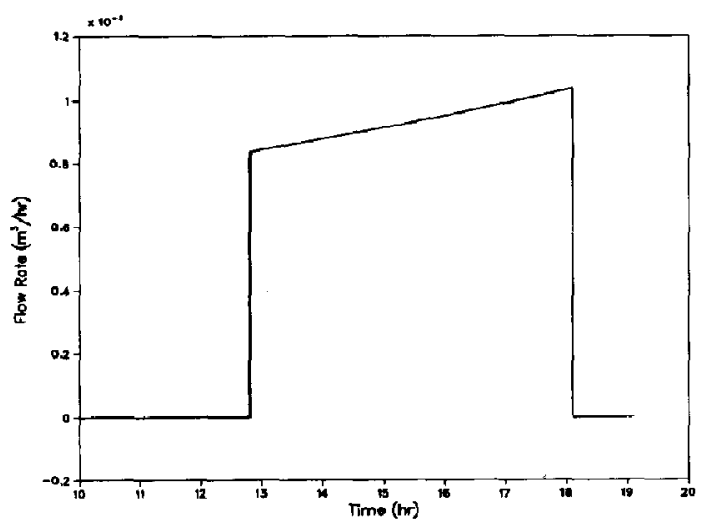

Fig. 8. Optimal input profile (example 3).

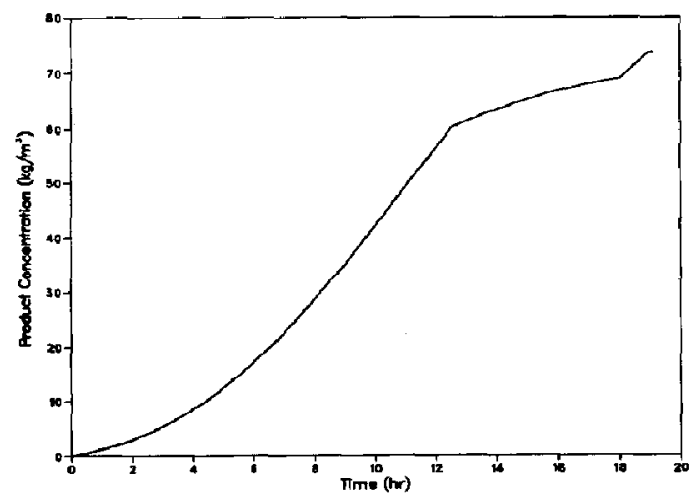

Fig. 9. Optimal time profile of ethanol (example 3).
Table 3(a). Model parameters

\begin{tabular}{lc}
\hline$K_{P}$ & $16.0 \mathrm{~kg} / \mathrm{m}^{3}$ \\
$K_{S}$ & $0.22 \mathrm{~kg} / \mathrm{m}^{3}$ \\
$K_{P}^{*}$ & $71.5 \mathrm{~kg} / \mathrm{m}^{3}$ \\
$K_{S}^{*}$ & $0.44 \mathrm{~kg} / \mathrm{m}^{3}$ \\
$Y$ & $0.1 \mathrm{~kg} / \mathrm{kg}^{*}$ \\
$S_{F}$ & $150.0 \mathrm{~kg} / \mathrm{m}^{3}$ \\
$t_{f}$ & $19.1 \mathrm{~h}$ \\
\hline
\end{tabular}

Table 3(b). Initial conditions

\begin{tabular}{lc}
\hline Case A & \\
\hline$X(0)$ & $1.0 \mathrm{~kg} / \mathrm{m}^{3}$ \\
$S(0)$ & $150.0 \mathrm{~kg} / \mathrm{m}^{3}$ \\
$P(0)$ & $0.0 \mathrm{~kg} / \mathrm{m}^{3}$ \\
$V(0)$ & $10.0 \times 10^{-3} \mathrm{~m}^{3}$ \\
\hline Case B & \\
\hline$X(0)$ & $0.9 \mathrm{~kg} / \mathrm{m}^{3}$ \\
$S(0)$ & $165.0 \mathrm{~kg} / \mathrm{m}^{3}$ \\
$P(0)$ & $0.0 \mathrm{~kg} / \mathrm{m}^{3}$ \\
$V(0)$ & $10.0 \times 10^{-3} \mathrm{~m}^{3}$ \\
\hline
\end{tabular}

Table 3(c). Sensitivity of error in switching time on performance index

\begin{tabular}{cc}
\hline $\begin{array}{c}\text { Assumed switching } \\
\text { time (h) }\end{array}$ & $\begin{array}{c}\text { Performance index } \\
\left(\mathrm{kg} / \mathrm{m}^{3}\right)\end{array}$ \\
\hline 12.00 & -73.607 \\
12.25 & -73.843 \\
12.50 & -73.850 \\
12.75 & -73.833 \\
13.00 & -73.749 \\
\hline
\end{tabular}

Table 3(d). Open-loop vs closed-loop implementation

Optimal solution for case A

Initial conditions: case $A$ in Table $3(b)$

Switching times: $12.5,18 \mathrm{~h}$

Ethanol concentration at final time: $73.850 \mathrm{~kg} / \mathrm{m}^{3}$

Optimal solution for case B

Initial conditions: case $R$ in Table $3(\mathrm{~b})$

Switching times: $14.5,17.5 \mathrm{~h}$

Ethanol concentration at final time: $79.660 \mathrm{~kg} / \mathrm{m}^{3}$

$$
\text { Open-loop implementation }
$$

Initial conditions: case $B$ in Table $3(\mathbf{b})$

Switching times: $12.5,18 \mathrm{~h}$ (from case A)

Input profile: optimal solution for case A

Ethanol concentration at final time: $73.408 \mathrm{~kg} / \mathrm{m}^{3}$

Closed-loop implementation

Initial conditions: case $B$ in Table 3(b)

Switching times: $12.5,18 \mathrm{~h}$ (from case A)

Input profile: state feedback law from eq. (70)

Ethanol concentration at final time: $78.049 \mathrm{~kg} / \mathrm{m}^{3}$ 
The sensitivity of the performance index to errors in the first switching time was checked. In the nonsingular regions $u$ was set to 0 . It was assumed that there was an error in estimating the first switching time. Assuming various values of the first switching time, the feeding profile was calculated using the static state feedback law in the singular region. It is observed [Table 3(c)] that the final performance index is quite robust to errors in the assumed switching time. A similar effect was obscrved for errors in the second switching time (results not shown). From this study, we conclude that the performance index is not very sensitive to errors in the switching times and so a priori estimates of the switching times can be used in closed-loop implementation. The sensitivity of yield optimization with respect to errors in the initial conditions was also studied. Feedback implementation was compared to the conventional open-loop implementation. The results are summarized in Table 3(d). It is observed that there is a reduction of about $10 \%$ in the performance index when we implement the optimal input profile in an open-loop fashion. However, there is a $6.32 \%$ improvement in the performance index over the open-loop scheme when the closed-loop scheme is used. Thus, the closed-loop scheme is able to attenuate errors in the initial conditions.

\section{Example 4: dynamic state feedhack}

We consider a yield optimization problem in a semi-batch chemical reactor, where the following reactions are occurring:

$$
\begin{aligned}
& A \rightarrow B \\
& B \rightarrow C \\
& A \rightarrow D .
\end{aligned}
$$

The reaction from $A$ to $B$ follows the following kinetics:

$$
-r=\frac{k_{1} C_{A} C_{B}}{k_{2}+k_{3} C_{A}+k_{4} C_{A}^{2}}
$$

All other reactions are first-order reactions. The unsteady-state mass balance equations are given by

$$
\begin{aligned}
\frac{\mathrm{d}\left(C_{A} V\right)}{\mathrm{d} t} & =-\frac{k_{1} C_{A} C_{B} V}{k_{2}+k_{3} C_{A}+k_{4} C_{A}^{2}}-k_{D} C_{A} V+s_{F} u \\
\frac{\mathrm{d}\left(C_{B} V\right)}{\mathrm{d} t} & =\frac{k_{1} C_{A} C_{B} V}{k_{2}+k_{3} C_{A}+k_{4} C_{A}^{2}}-k_{C} C_{B} V \\
\frac{\mathrm{d}\left(C_{D} V\right)}{\mathrm{d} t} & =k_{D} C_{A} V \\
\frac{\mathrm{d} V}{\mathrm{~d} t} & =u .
\end{aligned}
$$

The objective is to operate the reactor so that the total amount of $B$ is maximized at a prespecified final time $t_{f}$. Thus, the performance index to minimize is

$$
J=-\left.C_{B} V\right|_{t=t_{f}} \text {. }
$$

Define

$$
\begin{aligned}
& x_{1}=C_{A} V \\
& x_{2}=C_{B} V \\
& x_{3}=C_{D} V \\
& x_{4}=V .
\end{aligned}
$$

The optimization problem can be stated as follows. Minimize

$$
J=-x_{2}\left(t_{f}\right)
$$

subject to

$$
\begin{array}{r}
\frac{\mathrm{d}}{\mathrm{d} t}\left[\begin{array}{l}
x_{1} \\
x_{2} \\
x_{3} \\
x_{4}
\end{array}\right]=\left[\begin{array}{c}
-\frac{k_{1} x_{1} x_{2} x_{4}}{k_{2} x_{4}^{2}+k_{3} x_{1} x_{4}+k_{4} x_{1}^{2}}-k_{D} x_{1} \\
\frac{k_{1} x_{1} x_{2} x_{4}}{k_{2} x_{4}^{2}+k_{3} x_{1} x_{4}+k_{4} x_{1}^{2}}-k_{C} x_{2} \\
k_{D} x_{1} \\
0
\end{array}\right] \\
+\left[\begin{array}{c}
s_{F} \\
0 \\
0 \\
1
\end{array}\right] u
\end{array}
$$

The system model is of the form

$$
\dot{x}=f(x)+g(x) u
$$

where

$$
f(x)=\left[\begin{array}{c}
x=\left[\begin{array}{c}
x_{1} \\
x_{2} \\
x_{3} \\
x_{4}
\end{array}\right] \\
\frac{k_{1} x_{1} x_{2} x_{4}}{k_{2} x_{4}^{2}+k_{3} x_{1} x_{4}+k_{4} x_{1}^{2}}-k_{C} x_{2} \\
k_{D} x_{1} \\
0 \\
g(x)=\left[\begin{array}{c}
k_{1} x_{1} x_{4} x_{4} \\
s_{F} \\
0 \\
0 \\
1
\end{array}\right] .
\end{array}\right.
$$

It can be easily verified that the vector fields $g(x)$, $a d_{f}^{1} g(x), a d_{f}^{2} g(x), a d_{f}^{3} g(x)$ are linearly independent. and thus condition (9) is satisfied everywhere. For this 


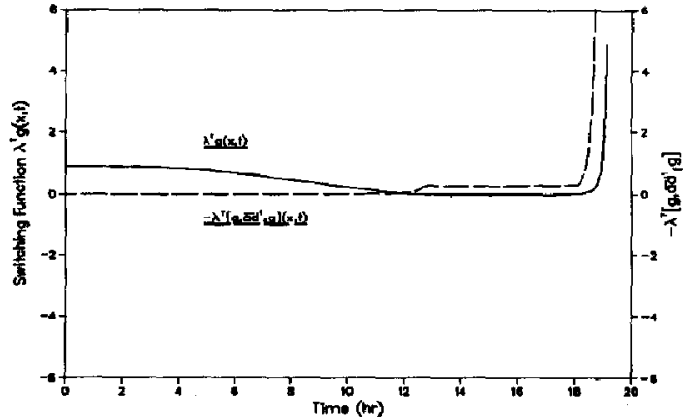

Fig. 10. Profile of switching function $\lambda^{T} g(x, t)$ and secondorder condition $-\lambda^{T}\left[g, \overline{a d}_{f}^{1} g\right](x, t)$ (example 3 ).

system $n=4$ and $s=1$. Thus, the optimal state feedback in the singular region will be dynamic. Substituting $n=4$ and $s=1$ in eq. (21), we obtain a dynamic feedback law by setting the determinant of

$g(x)$

$$
\begin{aligned}
& a d_{f}^{1} g(x) \\
& a d_{f}^{2} g(x)+\left[g, a d_{f}^{1} g\right](x) u \\
& a d_{f}^{3} g(x)+\left(\left[g, a d_{f}^{2} g\right](x)+\left[f,\left[g, a d_{f}^{1} g\right]\right](x)\right. \\
& \left.\quad+\left[g,\left[g, a d_{f}^{1} g\right]\right](x) u\right) u+\left[g, a d_{f}^{1} g\right](x) \frac{\mathrm{d} u}{\mathrm{~d} t}
\end{aligned}
$$

equal to zero. Since the reactor starts with a high concentration of $A$ and the reaction rate is a decreasing function of $A$, we expect that there will be an initial nonsingular phase where no $A$ is fed, so that the concentration of A decreases, followed by a singular phase which involves feeding of species $A$. The terminal conditions of the adjoint states $\lambda$ are compatible with the first-order necessary condition on the singular region, $\lambda^{T} g(x)=0$. Thus, intuitively we expect that there will be two phases-a nonsingular phase followed by a singular phase with one switching time to be calculated.

An initial estimate of the switching time was found by performing a one-dimensional numerical search as described by Lim et al. (1986). This estimate was further refined till the first-order condition $\lambda^{T} g(x)=0$ was satisfied on the singular region. Figures 11 and 12 show the optimal feeding profile and the optimal profile of species $B$ for the system parameters in Table 4(a) and the initial conditions in Table 4(b). Figure 13 depicts the corresponding time profiles of the switching function $\left(\lambda^{T} g(x)\right)$ and the second-order condition $\left(-\lambda^{T}\left[g, a d{ }_{f}^{1} g\right](x)\right)$. The switching function vanishes in the singular time interval and is positive everywhere else. The positivity of the switching function is consistent with $u(t)$ taking its minimum value $(u=0)$ in the nonsingular interval. Furthermore, the secondorder condition $-\lambda^{T}\left[g, \operatorname{ad}_{f}^{1} g\right](x, t) \geqslant 0$ is satisfied in the singular region and so the singular extremal is indeed optimal.

Since the optimal state feedback is dynamic, we

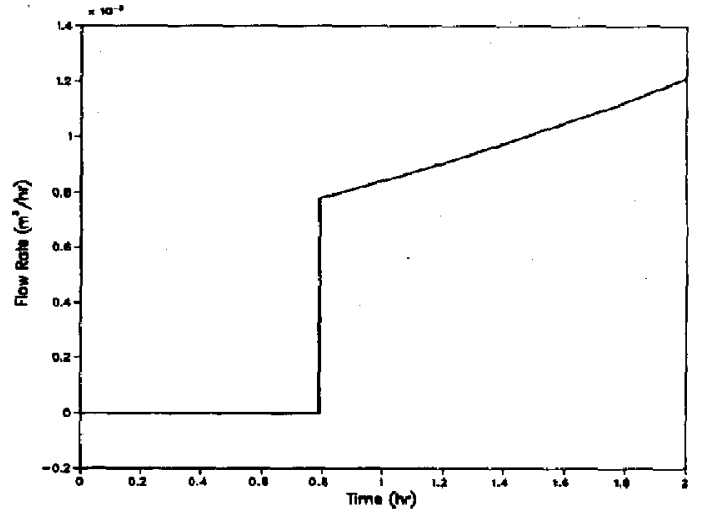

Fig. 11. Optimal input profile (example 4).

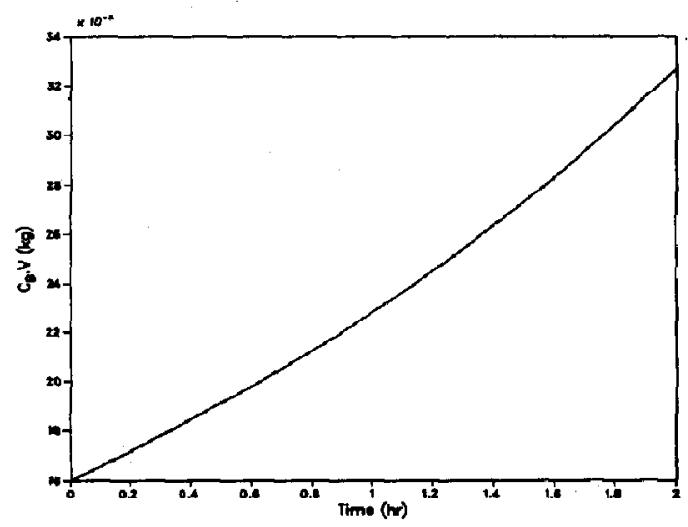

Fig. 12. Optimal time profile of species B (example 4).

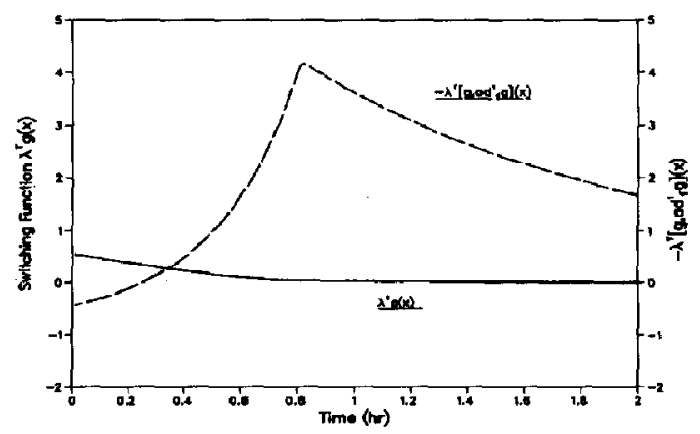

Fig. 13. Profile of switching function $\lambda^{T} g(x)$ and secondorder condition $-\lambda^{T}\left[g, a d_{f}^{1} g\right](x)$ (example 4).

need the initial condition of $u$ to implement the state feedback law. The effect of error in the initial condition of $u$ on the final performance index was studied. The results are shown in Table $4(\mathrm{c})$. It is observed that the final performance index is very robust to errors in the assumed initial condition for $u(t)$. From this study, we conclude that the performance index is not sensitive at all to errors in the initial condition for $u(t)$ and 
so an a priori estimate of the initial condition for $u(t)$ can be used in closed-loop implementation.

The robustness of the dynamic state feedback law with respect to errors in the system parameter $k_{1}$ was tested. With the initial condition for $u$ and switching time from the nominal case, the dynamic feedback law was implemented using a 13.4\% higher $k_{1}$ than its true value. The results are summarized in Table $4(d)$. It is observed that the performance index is very close to that obtained in the nominal case.

Acknowledgement-Financial support by NSF Research Grant BCS-8912627 is gratefully acknowledged.

Table 4(a). Model parameters

\begin{tabular}{ll}
\hline$k_{1}$ & $0.530 \mathrm{~m}^{3} / \mathrm{kmol} \mathrm{h}$ \\
$k_{2}$ & 1.00 \\
$k_{3}$ & $1.00 \mathrm{~m}^{3} / \mathrm{kmol}^{2}$ \\
$k_{4}$ & $1 / 22 \mathrm{~m}^{3} / \mathrm{kmol}^{2}$ \\
$k_{c}$ & $0.01 \mathrm{l} / \mathrm{h}$ \\
$k_{D}$ & $0.01 \mathrm{~h}$ \\
$t_{f}$ & $2.00 \mathrm{~h}$ \\
\hline
\end{tabular}

Table 4(b). Initial conditions

\begin{tabular}{ll}
\hline$C_{A}(0)$ & $7.5 \mathrm{~kg} / \mathrm{m}^{3}$ \\
$C_{B}(0)$ & $8.0 \mathrm{~kg} / \mathrm{m}^{3}$ \\
$C_{D}(0)$ & $0.0 \mathrm{~kg} / \mathrm{m}^{3}$ \\
$V(0)$ & $2.0 \times 10^{-3} \mathrm{~m}^{3}$ \\
\hline
\end{tabular}

Table 4(c). Sensitivity of error in initial condition of $u(t)$ on performance index

\begin{tabular}{cc}
\hline $\begin{array}{c}\text { Assumed initial } \\
\text { condition of } u(t)\end{array}$ & $\begin{array}{c}\text { Performance index } \\
(\mathbf{k g})\end{array}$ \\
\hline 0.00 & $-32.726 \times 10^{-3}$ \\
0.25 & $-32.726 \times 10^{-3}$ \\
0.50 & $-32.726 \times 10^{-3}$ \\
0.75 & $-32.726 \times 10^{-3}$ \\
1.00 & $-32.726 \times 10^{-3}$ \\
\hline
\end{tabular}

\section{NOTATION}

$C_{A}$ concentration of species $A, \mathrm{~kg} / \mathrm{m}^{3}$

$C_{B}$ concentration of species $B, \mathrm{~kg} / \mathrm{m}^{3}$

$C_{C}$ concentration of species $C, \mathrm{~kg} / \mathrm{m}^{3}$

$C_{D}$ concentration of species $\mathrm{D}, \mathrm{kg} / \mathrm{m}^{3}$

det determinant

$f \quad$ vector function in dynamic model (2) or (25)

$g$ vector function in dynamic model (2) or (25)

$H$ Hamiltonian

$H_{u} \quad$ first derivative of Hamiltonian with respect to $u$

$H_{u}$ second derivative of Hamiltonian with respect to $u$

$I \quad$ identity matrix

$J$ performance index

$k_{1} \quad$ kinetic parameter in eq. (71), $\mathrm{m}^{3} / \mathbf{k g ~ h}$

$k_{2} \quad$ kinetic parameter in eq. (71)

$k_{3} \quad$ kinetic parameter in eq. (71), $\mathrm{m}^{3} / \mathrm{kg}$

$k_{4} \quad$ kinetic parameter in eq. (71), $\mathrm{m}^{3} / \mathrm{kg}^{2}$

$k_{B} \quad$ kinetic rate constant of reaction $A \rightarrow B, 1 / h$

$k_{C} \quad$ kinetic rate constant of reaction $B \rightarrow C, 1 / h$

$k_{D} \quad$ kinetic rate constant of reaction $A \rightarrow D, 1 / h$

$K_{1}$ dissociation constant in the Haldane-Monod function [eq. (49)], $\mathrm{kg} / \mathrm{m}^{3}$

$K_{2}$ inhibition constant in the Haldane-Monod function [eq. (49)], $\mathrm{kg} / \mathrm{m}^{3}$

$K_{P} \quad$ kinetic parameter in rate eq. $(63), \mathrm{kg} / \mathrm{m}^{3}$

$K_{P}^{*} \quad$ kinetic parameter in rate eq. $(64), \mathrm{kg} / \mathrm{m}^{3}$

$K * \quad$ kinetic parameter in rate eq. $(64), \mathrm{kg} / \mathrm{m}^{3}$

$K_{s} \quad$ kinetic parameter in rate eq. $(63), \mathrm{kg} / \mathrm{m}^{3}$

$n$ number of state equations $(=$ dimension of state vector $x$ )

$P \quad$ product concentration, $\mathrm{kg} / \mathrm{m}^{3}$

$s \quad$ degree of singularity

$s_{F}$ concentration of feed, $\mathrm{kg} / \mathrm{m}^{3}$

$S$ substrate concentration, $\mathrm{kg} / \mathrm{m}^{3}$

$t$ time

$t_{f} \quad$ final time, $\mathrm{h}$

$u$ manipulated input

$V$ volume, $\mathrm{m}^{3}$

$x \quad$ vector of system states

$X$ cell mass concentration, $\mathrm{kg} / \mathrm{m}^{3}$

$y$ output

$Y \quad$ yield coefficient

\section{Greek letters}

$\beta$ adjustable parameter in the feedback law (19)

$\varepsilon \quad$ specific product formation rate, $1 / \mathrm{h}$

Table 4(d). Robustness analysis

\begin{tabular}{lll}
\hline Parameters in control law & Parameters in process & Performance index \\
\hline$k_{1}=0.530$ & $k_{1}=0.530$ & $-32.726 \times 10^{-3} \mathrm{~kg}$ \\
All other parameter & All other parameter \\
values from Table 4(a) & values from Table $4(\mathrm{a})$ & \\
$k_{1}=0.601$ & $k_{1}=0.530$ & $-32.715 \times 10^{-3} \mathrm{~kg}$ \\
All other parameter & All other parameter \\
values from Table 4(a) & values from Table 4(a) & \\
\hline
\end{tabular}


$\lambda$ vector of adjoint states

$\mu \quad$ specific growth rate, $1 / \mathrm{h}$

$\mu_{0} \quad$ kinetic parameter in rate eq. $(64), \mathrm{m}^{3} / \mathrm{kg} \mathrm{h}$

$\mu_{\mathrm{m}} \quad$ kinetic parameter in rate eq. (49), $\mathrm{m}^{3} / \mathrm{kg} \mathrm{h}$

$v_{0} \quad$ kinetic parameter in rate eq. (65), $\mathrm{m}^{3} / \mathrm{kg} \mathrm{h}$

$\phi \quad$ scalar function expressing the performance index in terms of the system states

\section{REFERENCES}

Aiba, S., Humphery, A. E. and Millis, N. F., 1973, Biochemical Engineering, p. 148. University of Tokyo Press, Tokyo.

Bailey, J. E. and Ollis, D. F., 1986, Biochemical Engineering Fundamentals. McGraw-Hill, New York.

Bryson, A. E. and Ho, Y, C, 1975, Applied Optimal Control. Wiley, New York.

Chen, S. A. and Jeng, W. F., 1978, Minimum end time policies for batchwise radical chain polymerization. Chem. Engng Sci. 33, 735.

Chen, S. A. and Huang, N. W., 1981, Minimum end time policies for batchwise radical chain polymerization-III. The initiator addition policies. Chem. Engng Sci. 36, 1295.

Denn, M. M., 1969, Optimization by Variational Methods. McGraw-Hill, New York.

Edwards, V. H., 1970, The influence of high substrate concentrations on microbial kinetics. Biotechnol. Bioengng 12, 679.

Ellis, M. F., Taylor, T. W., Gonzalez, V. and Jensen, K. F., 1988 , Estimation of the molecular weight distribution in batch polymerization. A.I.Ch.E. J. 34(8), 1341.

Fishman, V. M. and Biryukov, V. V., 1974, Kinetic model of secondary metabolite production and its use in computation optimal conditions. Biotechnol. Bioengng Symp. 4, 647.

Gabasov, R. and Kirillova, F. M., 1972, Higher order necessary conditions for optimality. SIAM J. Control 10(1), 127.

Hicks, J., Mohan, A. and Ray, W. H., 1969, The optimal control of polymerization reactors. Can. J. chem. Engng $47,590$.

Hunt, L. R, 1982, Sufficient conditions for controllability. IEEE Trans. Circuits Syst. CAS-29(5), 285.

Hunt, L. R., Su, R, and Meyer, G., 1983, Global transformations of nonlinear systems. IEEE Trans. Automat. Control AC-28, 24.

Jazwinski, A. H., 1970, Stochastic Processes and Filtering Theory. Academic Press, New York.

Kelley, H. J., 1964, A second variation test for singular extremals. $A I A A J .2(8), 1380$.

Kelley, H. J., Kopp, R. E. and Moyer, H. G., 1967, Singular extremals, in Topics in Optimization (Edited by G. Leitmann), p. 63. Academic Press, New York.

Kopp, R. E. and Moyer, H. G., 1965, Necessary conditions for singular extremals. $A I A A J .3,1439$.

Krener, A. J., 1977, The higher order maximal principle and its application to singular extremals. SIAM J. Control Optimization 15(2), 256.

Lamnabhi-Lagarrigue, F. and Stefani, G., 1990, Singular optimal control problems: on the necessary conditions of optimality. SIAM J. Control Optimization 28, 823.
Lewis, R. M., 1980, Definitions of order and junction conditions in singular optimal control problems. SIAM J. Control Optimization 18, 21.

Lim, H. C., Modak, J. M. and Bonte, P., 1986, Computational algorithms for optimal feed rates for a class of fedbatch fermentation: numerical results for penicellin and cell mass production. Biotechnol. Bioengng 28, 1408.

Ljung, L., 1985, Estimation of parameters in dynamical systems, in Handbook of Statistics (Edited by E. J. Hannan, P. R. Krishnaiah and M. M. Rao), Vol. 5. Elsevier, Amsterdam.

McDanell, J. P. and Powers, W. F., 1971, Necessary conditions for joining optimal singular and nonsingular subarcs. SIAM J. Control 9(2), 161.

Menawat, A., Muthurasan, R. and Coughanowr, D. R., 1987, Singular control strategy for a fed-batch bioreactor: numerical approach. A.I.Ch.E. J. 33, 776.

Miura, Y., Tsuchiya, K., Yamamoto, H. and Okazaki, M. 1975 , Kinetics of the adaptation process in the growth of Bacillus subtilis. J. Ferment. Technol. 53, 67.

Modak, J. M., 1988, A theoretical and experimental optimization of fed-batch fermentation processes. $\mathrm{Ph} . \mathrm{D}$. thesis, Purdue University, West Lafayette, IN.

Modak, J. M. and Lim, H. C., 1987, Feedback optimization of fed-batch fermentation. Biotechnol. Bivengng 30, 528 .

Ohno, H., Nakanishi, E. and Takamatsa, T., 1976, Optimal control of a semi-batch fermentation. Biotechnol. Bioengng, 18, 847.

Parulekar, S. J. and Lim, H. C, 1985, Modeling, optimization and control of semi-batch bioreactors. Adv. biochem. Engng Biotechnol. 32, 207.

Ray, W. H., 1981, Advanced Process Control. MeGraw-Hill, New York.

Robbins, H. M., 1967, A generalized Legendre condition for singular optimal control problems. Report No. SRRCRR-64-95, Sperry Rand Research Center, Sudbury, MA.

Rouff, $M$. and Lamnabhi-Lagarrigue, F., 1986, $A$ new approach to nonlinear optimal feedback law. Syst. Control Lett. 7, 411 .

Sacks, M. E., Lee, S. and Biesenberger, J. A., 1972, Optimal policies for batch, chain addition polymerizations. Chem. Engng Sci. 27, 2281.

Stephanopoulos, G. and San, K. Y., 1984, Studies on on-line bioreactor identification: I. Theory. Biotechnol. Bioengng 26, 1176 .

Schuler, H. and Papadopoulou, S., 1986, Real-time estimation of the chain-length distribution in a polymerization reactor: II. Comparison of estimated and measured distribution functions. Chem. Engng Sci. 41, 2681.

Staniskis, J. and Levisauskas, D., 1984, An adaptive control algorithm for fed-batch culture. Biotechnol. Bioengng 26, 419.

Thomas, I. M. and Kiparissides, C., 1984, Computation of near-optimal temperature and initiator policies for a batch polymerization reactor. Can. J. chem. Engng 62, 284.

Weigand, W. A., 1981, Maximum cell productivity by repeated fed-batch cultures for constant yield case. Biotechnol. Bioengng 23, 249.

Yamane, T. and Shimizu, S., 1984, Fed-batch techniques in microbial processes. Adv. biochem. Engng 30, 148. 